\title{
LEFSCHETZ TYPE PENCILS ON CONTACT MANIFOLDS*
}

\author{
F. PRESAS ${ }^{\dagger}$
}

\begin{abstract}
We define the concept of Lefschetz contact pencil and we show the existence of such structures on any contact manifold. The main idea of the proof is a generalization of the Donaldson's arguments used in the symplectic case. We will analyze some of the applications of this existence theorem for the topology of approximately holomorphic contact submanifolds. In particular, we study the topological relationship between the contact submanifolds constructed in [IMP99]. Lastly, we recover the notion of contact blow-up to get fibrations from the pencils imitating the symplectic case.
\end{abstract}

1. Introduction. S. Donaldson in [Do96] has adapted the concept of very ample bundle to the symplectic setting. Following this idea some results of complex projective geometry have been generalized to symplectic geometry, such as Bertini's theorem and Lefschetz's hyperplane theorem in [Do96], connectedness of the space of "good" sections of a very ample bundle in [Au97], divisors on projective fibrations in [Pa98], special position theorems in [Pa99], existence of Lefschetz pencils in [Do99], existence of branched coverings of symplectic 4-manifolds over $\mathbb{C P}^{2}$ and associated invariants [Au00, AK00], and Kodaira's embeddings theorems and symplectic determinantal submanifolds in [MPS99]. These ideas have opened a new insight in symplectic geometry allowing to understand symplectic manifolds through the study of the linear systems associated to a "very ample" vector bundle.

In [IMP99] the idea of [Do96] of working with approximately $J$-holomorphic sections was partially translated to the contact case. With these sections and a generalization of the local estimated transversality result of [Do96], which is the key of the symplectic approach, the ideas of [Do96, Au97] were translated word by word to the contact setting. The only important loss was the isotopy results which have been developed in the symplectic theory starting with the ideas of D. Auroux in [Au97]. The main goal of this paper is to show how to develop a contact geometry of linear systems analogous to the symplectic case. This will also show how to partially recover the isotopy results in the theory.

We will prove a theorem analogous to that of [Do99]. In fact, we will show the existence of a certain class of pencils on a contact manifold. The main tool in the proof will be a generalization of the local transversality theorem proved in [Do99]. As in the symplectic case this result is not strictly necessary for the proof, but it has interest in its own. It could be used to simplify the constructions of other linear systems, as for instance, the one in [MPS99] in the symplectic case.

In a sense our result is dual to the existence results of convex structures in contact manifolds which E. Giroux and J. P. Mohsen are developing by using asymptotically holomorphic techniques [GM01]. To explain this, it is worthy to go to the symplectic case. In that setting there are two different kinds of decompositions of a closed symplectic manifold. The first one consists of taking an asymptotically holomorphic divisor and its complementary. This complementary has been shown to be Stein [Bi01, GM01]. This can be thought as a kind of "convex decomposition" of the manifold.

*Received December 5, 2001; accepted for publication December 27, 2001.

†Departamento de Álgebra, Universidad Complutense de Madrid, 28040 Madrid, Spain (fpm@eucmos.sim.ucm.es). 
In the other hand it is possible to construct a Lefschetz pencil on the symplectic manifold. In [Do99] it is proved that any symplectic manifold admits a Lefschetz pencil decomposition. These two decompositions are not completely equivalent but they are very related. In fact, it is possible to pass from the Lefschetz pencil decomposition to the convex one.

In contact geometry, the construction that we are going to show must be understood like the analogue of the Donaldson's one. Moreover, what Giroux and Mohsen have proved is a convex decomposition in the sense of [Gi91], which is the analogue of Biran's symplectic construction. It would be interesting to relate our construction with this other one, like in the symplectic case. Some indications are given in the paper, specially in Section 6, but the equivalence is not absolutely understood.

A compatible chart in a contact manifold $(C, D)$ at a point $x$ will be a chart $\phi: U_{x} \subset C \rightarrow \mathbb{C}^{n} \times \mathbb{R}$, where $U_{x}$ is neighborhood of $x$, satisfying $\phi(x)=(0,0)$, $\left(\phi_{*}\right)(D(x))=\mathbb{C}^{n} \times\{0\}$ and moreover satisfying that the presymplectic form $\left(\phi_{*}\right) d \theta(x)$, when restricted to $\mathbb{C}^{n} \times\{0\}$, is a positive form of type $(1,1)$ at the origin of coordinates. If the contact manifold is exact we will impose also that $\left.\left\langle\phi_{*}(R)(x)\right), \frac{\partial}{\partial s}\right\rangle>0$ and say that the chart is oriented compatible, where $R$ is the Reeb vector field and $s$ is the real coordinate.

Definition 1.1. A (oriented) contact pencil on a closed (exact) contact manifold $C$ consists of the following data:

1. a codimension 4 contact submanifold $A \subset C$,

2. a finite set of smooth contact curves $\Delta=\bigcup_{i \in I} \gamma_{i} \subset C-A$,

3. a smooth map $f: V-A \rightarrow \mathbb{C P}^{1}$, whose restriction to the complementary of the set $\Delta$ is a submersion, satisfying also that $P_{\Delta}=f(\Delta)$ is a set of locally smooth curves with transversal self-intersections.

Also the data have to admit the following standard local models:

- At any point $a \in A$, there are (oriented) compatible coordinates $\left(z_{1}, \ldots, z_{n}, s\right) \in \mathbb{C}^{n} \times \mathbb{R}$ such that $A$ is locally given by $\left\{z_{1}, z_{2}=0\right\}$. And the function $f$ has the expression $f\left(z_{1}, \ldots, z_{n}, s\right)=\frac{z_{1}}{z_{2}} \in \mathbb{C P}^{1}$ near a.

- At a point $b_{i} \in \gamma_{i}$ there are (oriented) compatible coordinates in which $f$ is written as $f\left(b_{i}\right)+\varphi(s)+z_{1}^{2}+\ldots+z_{n}^{2}$, where $\varphi: \mathbb{R} \rightarrow \mathbb{C}$ satisfies $\varphi(0)=0$ and $\varphi^{\prime}(0) \neq 0$.

It is clear from the local model that the counter-image $f^{-1}(p)$ is a subset of $C-A$, whose closure in $C$ is smooth at $f^{-1}(p) \bigcap A$. Abusing language, we will call fiber over $b$ to the closure of the counter-image. It is a smooth submanifold if $b$ is a regular value of $f$. In other case we will have one or two singularities locally modelled by:

$$
\varphi(s)+z_{1}^{2}+\cdots+z_{n}^{2}
$$

In case $\operatorname{dim} C=3$, the smooth fibers will be (oriented) links on $C$. The link operation that is performed when the image crosses a circle $\gamma_{i}$ of the sphere $\mathbb{C P}^{1}$ looks, after general projection to a plane, as

The main result of this article is

THEOREM 1.2. Given a contact closed manifold $(C, D)$ (resp. exact) and $\alpha \in$ $H_{2 n-1}(C, \mathbb{R})$ which is reduction of an integer class, there exists a contact pencil on $C$ (resp. oriented) whose fibers are contact submanifolds, homologous to $\alpha$.

With this result at hand, it is easy to understand the possibility of finding general isotopic constructions for the contact submanifolds constructed in [IMP99]. Once fixed 


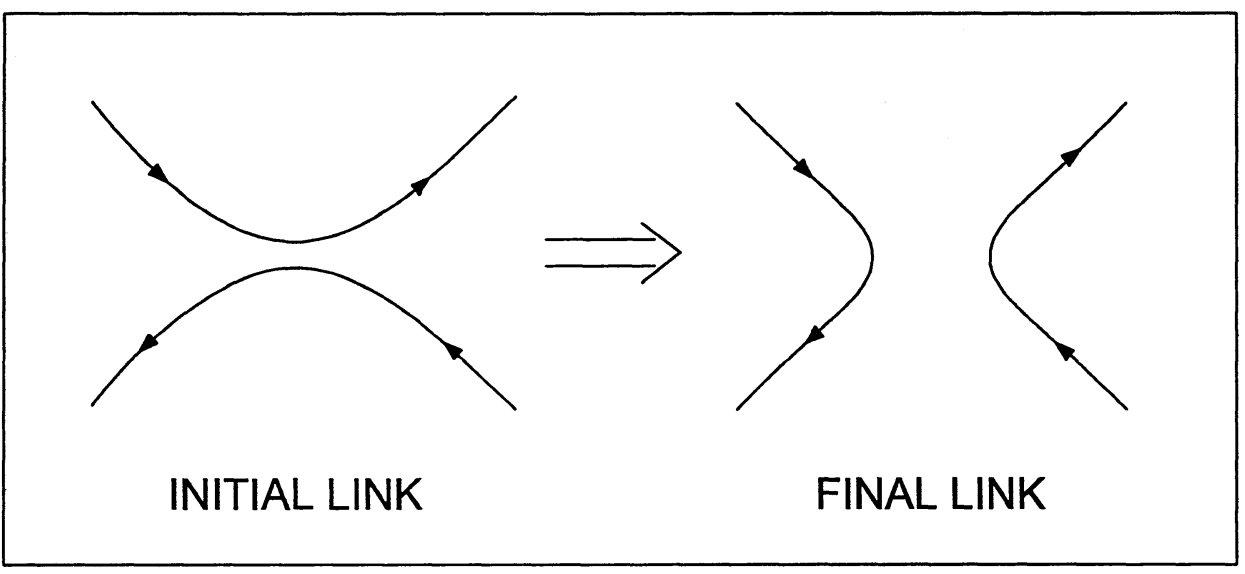

FIG. 1.1. Link operation

a compatible complex structure in the distribution, in Section 6 we will show how to define a sequence of contact fibrations $f_{k}$, satisfying $f_{k}(0) \simeq N_{k}$ and $f_{k}(\infty) \simeq N_{k}^{\prime}$, where $N_{k}$ and $N_{k}^{\prime}$ are sequences of codimension 2 contact submanifolds constructed with the method developed in [IMP99]. In order to assure the isotopy between $N_{k}$ and $N_{k}^{\prime}$ we have only to construct a path between 0 and $\infty$ in $\mathbb{C P}^{1}$ which does not intersect $\Delta$, because in this case $f$ restricted to the path is surjective. But this is only possible if 0 and $\infty$ are in the same connected component of $\mathbb{C P}^{1}-\Delta$, which is not true in general (contrarily to the symplectic case where $\Delta$ consists of isolated points). We will study in Subsection 6.1 the topological relationship between the counter-images of the points of a path crossing $\Delta$. This will prove that

$$
\begin{gathered}
H_{i}\left(N_{k}\right)=H_{i}\left(N_{k}^{\prime}\right), \\
\pi_{i}\left(N_{k}\right)=\pi_{i}\left(N_{k}^{\prime}\right),
\end{gathered}
$$

for $i=0, \ldots, n-2$. Moreover we will show how to make the result independent of the chosen complex structure and of the contact form, thus providing a verification of the "contact Lefschetz hyperplane theorem" proved in [IMP99]. The result brings us back to the Lefschetz original ideas to prove the hyperplane theorem (see [Le24]).

From a contact point of view we will check that all the fibers are related through surgeries along Legendrian spheres in the sense of [We91]. This will give some nice conjectures about the possible (and impossible) relations between the fibers. In particular, the related combinatorial descriptions of contact manifolds and the relation of contact structures with taut confoliations will be carried out in a forthcoming paper [IMP01].

In Section 2 we will give the basic results in contact geometry needed to develop the proof. In Section 3 we will give the proof of the exact case assuming transversality results of approximately holomorphic contact geometry. Afterwards, in Sections 4 and 5 , we develop the local approximately holomorphic techniques needed to achieve this transversality. In Section 6 we will study the relationship between approximately holomorphic sections transverse to $\mathbf{0}$. Later we adapt all the study to the non-exact case in Section 7. 
A brief discussion about contact blow-up is given in the last Section. There it is remarked the existence of the blow-up construction in some simple cases. Also a general proof will be given in a forthcoming paper [IMP01].

Acknowledgments: Special thanks to S. Donaldson by his kindness passing me the preprint [Do99] which has been absolutely fundamental to develop this work and by his useful observations about some of the parts of this paper. Also I thank D. Auroux and R. Paoletti for their help passing me their preprints. I have learnt Gromov's ideas about contact blow-up from Yasha Eliashberg. Finally I want to thank to the members of the GESTA ${ }^{1}$ seminar in Madrid for their support and interest through the elaboration of this work, specially Vicente Muñoz who has read and commented the previous versions of the document.

The author was conducting this research financed by the Ph.D. programs of Universidad Complutense de Madrid.

2. Definitions and results. We will assume along the proofs that $(C, D)$ is an exact contact manifold, where we have fixed a global contact form $\theta$. In Section 7 we will precise the changes needed to extend the results to the non-exact setting. We start recalling with the basic definitions and results of [IMP99].

2.1. Basic concepts. A complex structure on $C$ is a complex structure $J$ defined on $D$, interpreted as a symplectic vector bundle, compatible with the symplectic form $d \theta$. Recall that the distribution $D$ is contact, if and only if the restriction of $d \theta$ to $D$ is symplectic. The contact 1 -form defines a vector field $R$ by the conditions

$$
i_{R} \theta=1, \quad i_{R} d \theta=0,
$$

It is called the Reeb vector field. Given a complex structure we obtain a metric for the manifold

$$
g_{J}(u, v)=d \theta(u, J v)+\theta(u) \theta(v)
$$

This metric is called a contact metric. It depends on the fixed contact form. The $k$-rescaled contact metric will be defined as $g_{J, k}=k g_{J}$. If there is not risk of confusion we will denote simply $g_{k}$, supposing $J$ fixed along the proofs. (Observe that the $k$ rescaled contact metric $g_{k}$ is not the contact metric associated to $k^{\alpha} \theta$, for any $\alpha \in \mathbb{R}$ )

Now we give some definitions to control contact structures in $\mathbb{R}^{2 n+1}$.

Definition 2.1. The maximum angle between two subspaces $U, V \in \mathrm{Gr}_{\mathbb{R}}(r, n)$ is defined as:

$$
\angle_{M}(U, V)=\max _{u \in U} \angle(u, V)
$$

This angle defines a distance in the topological space $\operatorname{Gr}_{\mathbb{R}}(r, n)$ (for details see [MPS99]).

DEFINITION 2.2. Let $\theta_{k}$ be a sequence of contact forms in $\mathbb{R}^{2 n+1}$, with associated distributions $D_{k}=\operatorname{Ker} \theta_{k}$. The sequence is called c-asymptotically flat in the set $U \subset \mathbb{R}^{2 n+1}$ if

$$
\angle_{M}\left(D_{k}(0), D_{k}(x)\right) \leq c k^{-1 / 2}, \text { for all } x \in U
$$

\footnotetext{
${ }^{1}$ Geometría simpléctica con técnicas algebraicas.
} 
The sequence is called asymptotically flat if there exists some $c>0$ for which it is c-asymptotically flat.

If a contact distribution $D$ is defined at the origin by the horizontal subspace $\mathbb{R}^{2 n} \times\{0\} \subset \mathbb{R}^{2 n+1}$, we can define at a neighborhood of this point a canonical complex structure in $D$ by means of vertical projection of the canonical one defined on $\mathbb{R}^{2 n}$. This complex structure will be denoted $J_{0}$. In the case of the standard contact structure we can extend $J_{0}$ all over $\mathbb{R}^{2 n+1}$.

The standard contact structure in $\mathbb{R}^{2 n+1}$ is defined as $\theta_{0}=d s+\sum_{j=1}^{n} x_{j} d y_{j}$, where $\left(x_{j}, y_{j}, s\right) \in \mathbb{R}^{2 n+1}$. The sequence of metrics $\theta_{k^{1 / 2}}=k^{-1 / 2} d s+k^{-1} \sum x_{j} d y_{j}$, which is obtained from $\theta_{0}$ scaling the coordinates by a factor $k^{1 / 2}$, is a sequence of asymptotically flat contact forms on any bounded set of $\mathbb{R}^{2 n+1}$.

Following [IMP99] we can define $\partial$ and $\bar{\partial}$ operators in any kind of function, morphism or section defined on a contact manifold, restricting ourselves to the contact distribution, or equivalently projecting along the Reeb direction.

2.2. Approximately holomorphic geometry. We will talk about uniform constant, polynomial, when this constant, polynomial, etc. does not depend on the chosen point $x \in C$, nor in the integer $k$ appearing in the context. (However these constants can depend on the modulus of the given sections, on the nature of its derivatives, on the step of a recurrent reasoning, etc. but always independently of $k$ ).

We will use from [IMP99] the following

Lemma 2.3. Given $(C, \theta)$ a closed contact manifold, a point $x_{0} \in C$ and $J$ a compatible complex structure on Ker $\theta$, there exists a uniform constant $c>0$ and a contact Darboux chart $\psi:(C, \theta) \rightarrow\left(\mathbb{R}^{2 n+1}, \theta_{0}\right)$ satisfying that $\psi\left(x_{0}\right)=0$ and

$$
\frac{1}{2} g(v, w) \leq\left\langle\left(\psi_{*}\right)_{x} v,\left(\psi_{*}\right)_{x} w\right\rangle \leq 2 g(v, w), \forall x \in B_{g}\left(x_{0}, c\right), v, w \in T_{x} C .
$$

Also we have that $\left|\nabla^{r} \psi\right|=O(1)$ and $\left|\nabla^{r} \psi^{-1}\right|=O(1)$, for $r=1,2,3$. Moreover $|\bar{\partial} \psi(y)| \leq c^{\prime} d(x, y)$, for a uniform constant $c^{\prime}$.

A $k^{1 / 2}$-Darboux chart is a chart $\phi_{k}: B(x, \epsilon) \rightarrow \mathbb{R}^{2 n+1}$ such that $\left(\phi_{k}\right)_{*} \theta=\theta_{k^{1 / 2}}$. The following result is a direct corollary of Lemma 2.3:

Corollary 2.4. Given $(C, \theta)$ a closed contact manifold, a point $x_{0} \in C$ and $J$ a compatible complex structure on $\operatorname{Ker} \theta$, there exists a uniform constant $c>0$ such that there exists a contact chart $\psi:(C, \theta) \rightarrow\left(\mathbb{R}^{2 n+1}, \theta_{k^{1 / 2}}\right)$ satisfying that $\Psi\left(x_{0}\right)=0$ and

$$
\frac{1}{2} g_{k}(v, w) \leq\left\langle\left(\psi_{*}\right)_{x} v,\left(\psi_{*}\right)_{x} w\right\rangle \leq 2 g_{k}(v, w), \forall x \in B_{g_{k}}(x, c), v, w \in T_{x} C
$$

Also we have that $\left|\nabla^{r} \psi\right|=O(1)$ and $\left|\nabla^{r} \psi^{-1}\right|=O(1)$, for $r=1,2,3$. Moreover $\left|\nabla^{r} \bar{\partial} \psi(y)\right| \leq c^{\prime} k^{-1 / 2}$, for $r=0,1,2$, for a uniform constant $c^{\prime}$.

These results allows to locally trivialize contact manifolds in an approximately holomorphic way. The analogous notion of the $c$-bounds of [Do99] in the contact case is the following 
Definition 2.5. A sequence of sections $s_{k}$ of hermitian bundles $E_{k}$ over the contact manifold $(C, \theta)$ has mixed $C^{r}$-bounds $\left(c_{D}, c_{R}\right)$ at the point $x \in C$ if it satisfies

$$
\begin{aligned}
& \left|s_{k}(x)\right|<c_{D}, \\
& \left|\nabla_{D}^{j} s_{k}(x)\right|<c_{D}, \quad \forall j=1, \ldots, r . \\
& \left|\nabla^{j} s_{k}(x)\right|<c_{R}, \quad \forall j=1, \ldots, r . \\
& \left|\nabla^{j} \bar{\partial} s_{k}(x)\right|<c_{R} k^{-1 / 2}, \quad \forall j=0, \ldots, r-1 .
\end{aligned}
$$

The sequence has uniform mixed $C^{r}$-bounds $\left(c_{D}, c_{R}\right)$ if it satisfies these bounds at every point.

There we denote by $\nabla_{D}$ the restriction of the operator $\nabla$ to the subspace $D$. The metric used in the manifold $C$ to measure the norms in the precedent definition is the rescaled contact metric $g_{k}$. Recall that if $s_{k}^{j}$ has $\left(c_{D}^{j}, c_{R}^{j}\right)$ mixed $C^{r}$-bounds $(j=1,2)$, then $s_{k}^{1}+s_{k}^{2}$ has $\left(c_{D}^{1}+c_{D}^{2}, c_{R}^{1}+c_{R}^{2}\right)$ mixed $C^{r}$-bounds.

The following results are used to trivialize bundles over contact manifolds.

DEFINITION 2.6. A sequence of sections $s_{k}$ of bundles $E_{k}$ has mixed Gaussian decay in $C^{r}$-norm away from a point $x \in C$ if there exist a uniform polynomial $P$ and uniform constants $\lambda>0, c_{a}$ such that for all $y \in X$, the sequence $s_{k}(y)$ has mixed $C^{r}$-bounds

$$
\left(P\left(d_{k}(x, y)\right) \exp \left(-\lambda d_{k}(x, y)^{2}\right), c_{a} P\left(d_{k}(x, y)\right) \exp \left(-\lambda d_{k}(x, y)^{2}\right)\right) .
$$

Recall that the uniform constant $c_{a}$ is necessary because we will check afterwards that for different sequences of sections this constant cannot be fixed.

The prequantizable line bundle $L$ over an exact contact manifold $C$ is defined as the complex line bundle with connection such that $\operatorname{curv}(L)=-i d \theta$.

LEMMA 2.7 (Lemma 5 from [IMP99]). Let $(C, \theta)$ be a closed contact manifold. There exists a uniform constant $c_{s}>0$, such that given any point $x \in C$, there exists a sequence of sections $\sigma_{k, x}$ of $L^{\otimes k}$ satisfying $\left|\sigma_{k, x}\right| \geq c_{s}$ at every $y$ in a ball of $g_{k}$-radius 10 centered at $x$ and the sections $\sigma_{k, x}$ have uniform mixed Gaussian decay away from $x$ in $C^{3}$-norm (in this case $c_{a}=1$ ).

2.3. Transversality results. Following Donaldson [Do99] and Auroux [Au00] we set up the following definitions. A linear map $f: \mathbb{R}^{n} \rightarrow \mathbb{R}^{r}$ is $\eta$-transverse if it has a right inverse $\nu: \mathbb{R}^{r} \rightarrow \mathbb{R}^{n}$ such that $|\nu| \leq \eta^{-1}$. In the non-linear case we will say that $f: U \subset \mathbb{R}^{n} \rightarrow \mathbb{R}^{r}$ is $\eta$-transverse to $y \in \mathbb{R}^{r}$ over $U$ if $\forall x \in U$, such that $|f(x)-y|<\eta$, then $d f$ is $\eta$-transverse. Recall that this is an open condition. In fact, if $|f-g|_{C^{1}, U}<\epsilon / 10$ and $f$ is $\epsilon$-transverse to $y$ over $U$ then $g$ is, say, $\epsilon / 2$-transverse to $y$ in $U$.

The definition of transversality to $\mathbf{0}$ for sections of hermitian bundles over Riemannian manifolds is totally analogous. In the case of contact manifolds we have to strengthen the conditions.

DEFINITION 2.8. A section $s_{k}$ of the hermitian vector bundle $E_{k}$ over the contact manifold $(C, \theta)$ is $\eta$-transverse to $\mathbf{0}$ on $U \subset C$ if for all $x \in U$ such that $\left|s_{k}(x)\right|<\eta$ then $\nabla_{D} s_{k}(x)$ is $\eta$-transverse to 0 (with respect to the $g_{k}$-metric in $C$ ).

From the discussion of [IMP99] it follows that a sequence of sections $s_{k}$ of the bundles $E_{k}$ over the contact manifold $(C, \theta)$, which has uniform $\left(c_{D}, c_{R}\right)$ bounds and which is $\eta$-transverse to $\mathbf{0}$, has as zero set a contact submanifold, for $k$ large enough. The precise result of [IMP99] is 
TheOREM 2.9. Given a closed exact contact manifold $(C, \theta)$. Let $\epsilon>0$ and let $s_{k}$ be a sequence of sections of the bundles $E \otimes L^{\otimes k}$, for a fixed hermitian bundle $E$, with uniform mixed $C^{r}$-bounds $\left(c_{D}, c_{R}\right)$. Then there exists a real number $\eta>0$ (depending on $\epsilon, c_{D}$ and $c_{R}$ ), and a sequence $\sigma_{k}$ such that, for $k$ large enough:

1. $\sigma_{k}-s_{k}$ has mixed $C^{r}$-bounds $\left(\epsilon, c_{R}^{\prime}\right)$.

2. $\sigma_{k}$ is $\eta$-transverse to $\mathbf{0}$.

In [IMP99] the proof is developed for sequences with mixed $C^{2}$-bounds, but there is not any problem in generalizing it to the mixed $C^{r}$-bounds case. We need in this article mixed $C^{3}$-bounds. Given a section $s_{k}=\left(s_{k}^{0}, s_{k}^{1}\right)$ of the bundle $\mathbb{C}^{2} \otimes S \otimes L^{\otimes k}$, for a fixed hermitian line bundle $S$, whose zero set is $Z\left(s_{k}\right)$, we denote $F^{s_{k}}=\frac{s_{k}^{1}}{s_{k}^{0}}$ : $C-Z\left(s_{k}\right) \rightarrow \mathbb{C P}^{1}$ the projectivization of the section. The holomorphic part of the differential of this map will be denoted by $\partial F^{s_{k}}$. Now we state a generalization of Theorem 2.9 which will be proved in Section 4:

THEOREM 2.10. Given a closed exact contact manifold $(C, \theta)$. Let $\epsilon>0$ and let $s_{k}=\left(s_{k}^{0}, s_{k}^{1}\right)$ be a sequence of sections of the bundles $\mathbb{C}^{2} \otimes S \otimes L^{\otimes k}$ with mixed $C^{3}$-bounds $\left(c_{D}, c_{R}\right)$. Suppose that $s_{k}^{0}$ and $s_{k}$ are both transverse to $\mathbf{0}$. Then there exists $\eta>0$ (depending on $\epsilon, c_{D}$ and $c_{R}$ ), and a sequence $\sigma_{k}$ satisfying for $k$ large enough

1. $\sigma_{k}-s_{k}$ has mixed $C^{3}$-bounds $\left(\epsilon, c_{R}^{\prime}\right)$,

2. $\sigma_{k}^{0}$ and $\sigma_{k}^{0} \oplus \sigma_{k}^{1}$ are $\eta$-transverse to $\mathbf{0}$,

3. $\partial F_{k}^{\sigma}$ is $\eta$-transverse to 0 away from $Z\left(\sigma_{k}^{0}\right)$.

The techniques used in Section 4 improve slightly the ones of [IMP99] and thus could allow a simpler proof of Theorem 2.9 avoiding some of the complications of the globalization process in that article.

3. Proof of the main result. Take a complex line bundle $S$ with connection $\nabla$ satisfying that $\operatorname{curv}(\nabla)=P D(\alpha)$. This is possible since $\alpha$ is an integer class. Starting with any sequence of sections $s_{k}^{\prime}$ of $\mathbb{C}^{2} \otimes S \otimes L^{\otimes k}$ with mixed $C^{3}$-bounds, we can perturb it using Theorems 2.9 and 2.10 to achieve a sequence of sections $s_{k}$ verifying properties 2 and 3 of Theorem 2.10 and with mixed $C^{3}$-bounds $\left(c_{D}, c_{R}\right)$. We consider this sequence as starting datum and will use it to construct the oriented contact pencil.

From the $\eta$-transversality of $s_{k}$ the zero set $A=Z\left(s_{k}\right)$ is a codimension 4 contact manifold where $F^{s_{k}}$ is not well defined. We will write $F$ instead of $F^{s_{k}}$ whenever it causes no confusion. Now we will study, as in [Do99], the shape of the "bad set" $\Gamma=\{x \in C:|\partial F| \leq|\bar{\partial} F|\}$. From [IMP99] we know that if we prove that $\Gamma=\left\{x \in C: d_{D} F=0\right\}$ we will have obtained that the fibers of $F$ are contact at all smooth points. This will be proved in several steps.

Lemma 3.1. There is a constant $\xi>0$, depending only on $c_{D}, c_{R}$ and $\epsilon$, such that if $k$ is large enough then $\left|s_{0}\right| \geq \xi$ on $\Gamma$.

Proof. The proof is analogous to that of Lemma 7 in [Do99].

Define $\Delta$ as the set of points where $\partial F=0$. The connected components of $\Delta$ form a discrete set of smooth curves by the transversality condition imposed to $\partial F$. Also we can assure that this set of components is finite because it is contained in $\Gamma$, which by Lemma 3.1 is contained in the complementary of a $\gamma$-neighborhood of $W_{\infty}=Z\left(s_{k}^{0}\right)$, for $\gamma>0$ a uniform constant small enough. We define:

$$
\Omega_{\xi}=\left\{p \in C,\left|s_{0}(p)\right|>\xi / 2\right\} .
$$


The following step, adapting again Donaldson's argument, is to estimate the shape of the set $\Delta$. This is the content of the following:

PROPOSITION 3.2. There is a uniform constant $\rho_{0}>0$, such that the $\rho_{0}$ neighborhoods of each connected component $\gamma_{i}$ of $\Delta$ are disjoint and are contained in $\Omega_{\xi}$. Moreover for any $\rho<\rho_{0}$, for $k$ large enough (only depending on $\rho$ ), the set $\Gamma$ is contained in a $\rho$-neighborhood of the set $\Delta$.

The proof of this result is absolutely analogous to the proof of Proposition 9 in [Do99] and depends strongly on Lemma 8 in that paper. We refer the reader to [Do99] for the argument.

Finally, we need to perturb the sequence $\partial F^{s_{k}}$ in arbitrarily small neighborhoods of $A$ and $\Delta$ to achieve the local models required in Definition 1.1. The perturbation required in $\Delta$ needs a careful analysis, but again the situation in $A$ is a straightforward generalization of [Do99]. We need only to define

$$
L D_{x}=\nabla s_{0}^{k} \oplus \nabla s_{1}^{k}: T C_{x} \rightarrow L_{x}^{\otimes k} \oplus L_{x}^{\otimes k} .
$$

With this notation the result we need in our case is

Lemma 3.3. For a point $x \in A, F$ can be represented in the standard model of Definition 1.1 at $x$ if and only if $T A_{x} \cap D$ is a symplectic subspace and the restriction of $d \theta$ to the symplectic orthogonal $C A_{x}$ (in $D$ ) of $T A_{x} \cap D$ is a positive form of type $(1,1)$ with respect to the complex structure on $C A_{x}$ induced by $L D_{x}$.

We do not provide a proof of this Lemma, which follows word by word the proof of Lemma 11 in [Do99]. Thanks to the $\left(c_{D}^{\prime}, c_{R}^{\prime}\right)$ mixed $C^{3}$-bounds and the transversality of the sequence $s_{k}$, it is easy to check that a small $C^{3}$-perturbation of the sequence satisfies the hypothesis of Lemma 3.3, thus completing the study in the neighborhood of $A$.

Now we study the map $F$ near $\Delta$. Again Donaldson's ideas work in this case, however the adaptation of the proof needs some changes. Select a smooth connected curve $\gamma_{i}$ in $\Delta$. We are going to perturb $F$ in a $\gamma$-neighborhood of $\Delta$. By Lemma 3.2, the perturbations can be made in each connected component $\gamma_{i}$ in an independent way.

Recall that, for $k$ large enough, the curve $\gamma_{i}$ is contact, i.e. $\forall x \in \gamma_{i}, T_{x} C=$ $T_{x} \gamma_{i} \oplus D_{x}$. Moreover, the angle between $T_{x} \gamma_{i}$ and $D_{x}$ is bounded below by a uniform constant because of the transversality of the sequence. Using the contact metric $g_{J}$ associated to the fixed complex structure $J$ to define a geodesic flow, we can obtain a diffeomorphism:

$$
\phi_{i}: U_{\rho} \rightarrow V_{\rho} \subset S^{1} \times \mathbb{C}^{n}
$$

where $U_{\rho}$ is the $\rho$-neighborhood of $\gamma_{i}$ (in $g_{k}$ metric) and $V_{\rho}$ is its image by the flow, which is an open neighborhood of $S^{1} \times\{0\}$. We can construct a metric in $S^{1}$ by imposing the condition that $\left(\phi_{i}\right)_{\mid \gamma_{i}}$ is an isometry with respect to the rescaled contact metric $g_{k}$. In $\mathbb{C}^{n}$ we will fix the standard metric. The product metric will be denoted by $g_{0}^{k}$. We can select with this choice a uniform $\rho>0$ such that

$$
\lambda_{m} g_{k}\left(d \phi_{i}(v), d \phi_{i}(w)\right) \leq\left|g_{0}^{k}(v, w)\right| \leq \lambda_{M} g_{k}\left(d \phi_{i}(v), d \phi_{i}(w)\right), \quad \forall v, w \in T_{x} C, \forall x \in U_{\rho},
$$

where $\lambda_{m}, \lambda_{M}>0$ are uniform constants. Once we have fixed $\phi_{i}$, we obtain a distribution $D_{k}$ in $\phi_{i}\left(U_{\rho}\right)$ constructed as the image of the distribution $D$. Denote by 
$D_{h}$ the integrable distribution given as $\{p\} \times \mathbb{C}^{n}$ defined in $S^{1} \times \mathbb{C}^{n}$. Perhaps after shrinking $\rho$ uniformly, we can check that

$$
\angle_{M}\left(D_{k}(s, z), D_{h}(s, z)\right)<c_{u}|z| k^{-1 / 2}, \forall(s, z) \in V_{\rho} \subset S^{1} \times \mathbb{C}^{n},
$$

where $c_{u}>0$ is a uniform constant. Moreover, we can impose without loss of generality that $\left(\phi_{i}\right)_{*} J\left(\gamma_{i}\right)=J_{0}$. So we can project orthogonally $\left(\phi_{i}\right)_{*} J$ (defined on $D_{k}$ ) to $D_{h}$ obtaining a new almost complex structure $\hat{J}$. In fact it is easy to check, as in [Do96], that

$$
\partial=\partial_{0}+\bar{\mu} \bar{\partial}_{0}, \quad \bar{\partial}=\bar{\partial}_{0}+\mu \partial_{0}
$$

where $\partial$ and $\partial_{0}$ are the operators defined by the structures $\hat{J}$ and $J_{0}$ in $S^{1} \times \mathbb{C}^{n}$, and $|\mu(z)| \leq c|z| k^{-1 / 2}$, where $c$ is a uniform constant. In order to finish the proof we follow these steps: we will define the perturbation, afterwards we will prove it satisfies the conditions for the distribution $D_{h}$ with the almost complex structure $\hat{J}$, and finally we will check the result for the distribution $D_{k}$.

Given any differentiable function $f: C \rightarrow \mathbb{C}$, we denote $f_{0}=f \circ \phi_{i}^{-1}$. By the inequalities (3.1) we can use $F_{0}$ instead of $F$ for all the computations using the induced distribution $D_{k}$. To construct the perturbation we define the complex Hessian $H=\frac{1}{2} \partial \partial F$. Using the trivialization $\phi_{i}$ we may regard it as

$$
H_{0}(s, z)=\sum H_{\alpha \beta}(s) z_{\alpha} z_{\beta}
$$

on $\phi_{i}\left(U_{\rho}\right)$. Also we take a cut-off function $\beta_{\rho}: S^{1} \times \mathbb{C}^{n} \rightarrow[0,1]$ satisfying

1. $\beta_{\rho}\left(\phi_{i}(p)\right)=1$, if $d_{k}\left(p, \gamma_{i}\right) \leq \frac{\rho}{2}$.

2. $\beta_{\rho}\left(\phi_{i}(p)\right)=0$, if $d_{k}\left(p, \gamma_{i}\right) \geq \rho$.

3. $\left|\nabla \beta_{\rho}\right|=O\left(\rho^{-1}\right)$.

We can adjust $\beta$ to assure condition 3 because of equation (3.1). The constant $\rho<\rho_{0}$ will be fixed along the proof to assure that the conditions are satisfied (namely we will have to shrink $\rho$ in a uniform way). A modification of $F$ will be

$$
f_{0}(s, z)=\beta_{\rho}\left(w^{\prime}(s)+H_{0}(s, z)\right)+\left(1-\beta_{\rho}\right) F_{0}(s, z),
$$

where $w^{\prime}: S^{1} \rightarrow \mathbb{C}$ is any smooth function.

We denote by $\partial$ and $\bar{\partial}$ the operators associated to the almost complex structure $\hat{J}$ acting on $D_{h}$. Respectively we denote by $\partial_{k}$ and $\bar{\partial}_{k}$ the operators associated to the action of $\left(\phi_{i}\right)_{*} J$ in $D_{k}$. The equivalent to Lemma 10 of [Do99] is

LEMMA 3.4. If $\rho>0$ is small enough, $k$ is sufficiently large, $\left|w^{\prime}(s)-F_{0}(s, 0)\right|$ is sufficiently small and $\left|\frac{d w^{\prime}(s)}{d s}\right|=O(1)$, then the inequality $\left|\partial_{k} f_{0}\right| \leq\left|\bar{\partial}_{k} f_{0}\right|$ is only satisfied in $\gamma_{i}$.

Proof. First, assume that we are at a point where $\beta_{\rho}=1$. Then $f_{0}=w^{\prime}+H_{0}$ and

$$
\partial f_{0}=\partial H_{0}, \quad \bar{\partial} f_{0}=\bar{\partial} H_{0}
$$

The $\eta$-transversality of $\partial F_{0}$ yields the bound

$$
\left|\partial H_{0}(s, z)\right| \geq \eta|z|-\left|\vec{\partial}\left(\partial F_{0}\right)_{(z=0)}\right||z| .
$$


Now recall that $\bar{\partial} \partial+\partial \bar{\partial}=0$ on functions and that the norm of $\partial \bar{\partial} F_{0}$ is controlled because $F$ has uniform mixed $C^{3}$-bounds. Then we can write

$$
\left|\partial H_{0}(s, z)\right| \geq \eta|z|-c_{u} k^{-1 / 2}|z|
$$

Using the inequality (3.2) and that $\left|\frac{\partial}{\partial s} H_{\alpha \beta}(s)\right|=O(1)$ (as follows from the mixed $C^{3}$-bounds of $F$ ) and recalling the bound $\left|\frac{d w^{\prime}}{d s}\right|=O(1)$, we rewrite the inequality as

$$
\left|\partial_{k} H_{0}(s, z)\right| \geq \eta|z|-c_{u}^{\prime} k^{-1 / 2}|z|
$$

where $c_{u}^{\prime}>0$ is a uniform constant. On the other hand,

$$
\left|\bar{\partial} H_{0}\right| \leq c_{u}|z|^{2} k^{-1 / 2}
$$

and thus, by an analogous argument,

$$
\left|\bar{\partial}_{k} H_{0}\right| \leq c_{u}^{\prime \prime}|z|^{2} k^{-1 / 2}+c_{u}^{\prime \prime}|z| k^{-1 / 2} .
$$

Now, if we impose that $\left|\partial_{k} H_{0}\right| \leq\left|\bar{\partial}_{k} H_{0}\right|$, we obtain by comparing (3.4) and (3.5), $z=0$ for $k$ large enough.

Now we study points in the annulus containing the support of $\nabla \beta_{\rho}$. In this case,

$$
\bar{\partial} f_{0}=\bar{\partial} \beta_{\rho}\left(w^{\prime}+H_{0}-F_{0}\right)+\beta_{\rho} \bar{\partial} H_{0}+\left(1-\beta_{\rho}\right) \bar{\partial} F_{0} .
$$

Bounding the right hand side as in [Do99] we obtain an expression for the value $\left|\bar{\partial} f_{0}\right|$. Using (3.2) and the bounds $\left|\frac{d w^{\prime}}{d s}\right|=O(1)$ and $\left|\bar{\partial}_{k} F_{0}\right|=O\left(k^{-1 / 2}\right)$, we conclude

$$
\left|\bar{\partial}_{k} f_{0}\right| \leq c\left(\rho^{2}+k^{-1 / 2}+\left|F_{0}(s, 0)-w^{\prime}\right| \rho^{-1}\right) \text {. }
$$

In the same way we know that

$$
\partial_{k} f_{0}=\partial_{k} \beta_{\rho}\left(w^{\prime}+H_{0}-F_{0}\right)+\beta_{\rho} \partial_{k} H_{0}+\left(1-\beta_{\rho}\right) \partial_{k} F_{0} .
$$

Using the transversality of $F$ we can obtain a lower bound for $\left|\partial_{k} f_{0}\right|$. The argument follows the one of Donaldson arriving to the final expression

$$
\left|\partial_{k} f_{0}\right|-\left|\bar{\partial}_{k} f_{0}\right| \geq \frac{\eta \rho}{2}-c\left(\rho^{2}+k^{-1 / 2}+\left|w^{\prime}-F_{0}(s, 0)\right| \rho^{-1}\right)
$$

Obviously, once fixed a sufficiently small $\rho$, for $k$ large enough and $\left|F_{0}(s, 0)-w^{\prime}\right|$ small enough compared with $\rho$ the inequality is strictly positive for any point in the annulus.

To finish the proof we only have to check that the function $f$ conforms the local models at any point of $\Delta$. We use the perturbation $w^{\prime}$ to assure that the curves $\gamma_{i}$ project smoothly into $\mathbb{C P}^{1}$, this is equivalent to impose

$$
\nabla_{v} f(x) \neq 0
$$

for any point $x \in \Delta$ and any nonzero $v \in T_{x} \Delta$. This can be achieved by a generic perturbation, and so $w^{\prime}$ can be selected to get it. Also we can assure, using this perturbation, that the intersections of branches of $f(\Delta)$ are transverse, again by a genericity argument. 
Finally, given any $x \in \Delta$ there exists coordinates $\left(s, x_{1}, y_{1}, \ldots, x_{n}, y_{n}\right)$ such that $f$ is locally written as

$$
f\left(s, z_{1}, \ldots, z_{n}\right)=\varphi(s)+\sum H_{\alpha \beta}(s) z_{\alpha} z_{\beta},
$$

where $\varphi^{\prime}(0) \neq 0$ (this condition is equivalent to the smoothness of the branches of $f(\Delta))$. Now at a neighborhood of $s=0$ the 1-parametric family $\left(H_{\alpha \beta}(s)\right)$ of bilinear complex forms can be diagonalized by a smooth family of complex changes of coordinates if the eigenvalues of $\left(H_{\alpha \beta}\right)$ are all distinct. This is a genericity condition that can be achieved at all the points of $\Delta$ by a generic perturbation of $O\left(k^{-1 / 2}\right)$ in $F$, before starting the perturbation process which we have developed along this section. With this condition, we obtain a smooth family of invertible complex matrix $P(s)$ such that

$$
\sum H_{\alpha \beta}(s) z_{\alpha} z_{\beta}=z^{T} P(s)^{T} P(s) z
$$

where $z=\left(z_{1}, \ldots, z_{n}\right)$. Therefore the change of coordinates

$$
\begin{aligned}
\mathbb{R} \times \mathbb{C}^{n} & \rightarrow \mathbb{R} \times \mathbb{C}^{n} \\
(s, z) & \rightarrow(s, P(s) z)
\end{aligned}
$$

gives us the required local model. This finishes the proof of the main theorem.

4. Transversality results. In this section we will prove Theorem 2.10.

4.1. The globalization scheme. First we recall how the globalization process developed in [Do96] adapts to the contact setting. This adaptation was carried out in [IMP99]. Now, we set up the process in a functorial way in the style of [Au00]. As uaual, we denote by $(C, \theta)$ an exact contact manifold.

Definition 4.1. A family of properties $\mathcal{P}(\epsilon, x)_{x \in C, \epsilon>0}$ of sections of bundles over $C$ is local and mixed $C^{r}$-open if, given a section $s$ satisfying $\mathcal{P}(\epsilon, x)$, any section $\sigma$ such that $s-\sigma$ has $\left(\eta, c_{R}\right)$ at $x$ mixed $C^{r}$-bounds satisfies $\mathcal{P}\left(\epsilon-c_{u} \eta, x\right)$, for some constant $c_{u}$.

Proposition 4.2 ([IMP99]). Let $\mathcal{P}(\epsilon, x)_{x \in C, \epsilon>0}$ be a local and mixed $C^{r}$-open family of properties of sections of vector bundles $E_{k}$ over $C$. Assume there exist uniform constants $c, c^{\prime}, c^{\prime \prime}, p$ and a function $f: \mathbb{R}^{3} \rightarrow \mathbb{R}^{+}$such that, given any $x \in C$, any small enough $\delta>0$, and mixed $C^{r}$-bounded sections $s_{k}$ of $E_{k}$ with uniform mixed $C^{r}$-bounds, say $\left(c_{D}, c_{R}\right)$, there exist, for all large enough $k$, mixed $C^{r}$-bounded sections $\tau_{k, x}$ of $E_{k}$ with the following properties:

1. $\tau_{k, x}$ has mixed $C^{r}$-bounds $\left(c^{\prime} \delta, f\left(c^{\prime} \delta, c_{D}, c_{R}\right)\right)$,

2. the sections $\frac{1}{\delta} \tau_{k, x}$ have mixed Gaussian decay away from $x$ in $C^{r}$-norm,

3. $s_{k}+\tau_{k, x}$ satisfy the property $\mathcal{P}(\eta, y)$ for all $y \in B_{g_{k}}(x, c)$, with $\eta=$ $c^{\prime \prime} \delta\left(\log \left(\delta^{-1}\right)\right)^{-p}$.

Then, given any $\alpha>0$ and mixed $C^{r}$-bounded sections $s_{k}$ of $E_{k}$, there exist, for all large enough $k$, mixed $C^{r}$-bounded sections $\sigma_{k}$ of $E_{k}$, such that $s_{k}-\sigma_{k}$ has mixed $C^{r}$-bounds $\left(\alpha, c_{R}\right)$ for some $c_{R}>0$. Also, the sections $\sigma_{k}$ satisfy $\mathcal{P}(\epsilon, x)$ for some uniform $\epsilon>0$ at any $x \in C$.

Sketch of the proof. Although this is just a slight variation of the globalization argument in [IMP99] we provide the main lines by completeness. Let $S$ be a finite set of points in $C$ verifying the following properties: 
1. $\bigcup_{x \in S} B_{g_{k}}(x, c) \supset C$.

2. There exists a partition $S=\bigcup_{j \in J} S_{j}$ verifying that $d_{g_{k}}(x, y)>\mathcal{N}$ if $x, y \in S_{j}$. $\mathcal{N}$ will be fixed along the proof.

3. The cardinal of $J$ is $O\left(\mathcal{N}^{2 n+1}\right)$.

The idea is to achieve the property $\mathcal{P}(\epsilon, x)$ in all the balls $B_{g_{k}}(x, c), x \in S_{j}$ at once. Using the hypothesis at each $x \in S_{j}$ we can build a local section $\tau_{k, x}$ which satisfies $\mathcal{P}(\eta, y)$ for all $y \in B_{g_{k}}(x, c)$, where $\eta=c^{\prime \prime} \delta\left(\log \left(\delta^{-1}\right)\right)^{-p}$. We select $\mathcal{N}$ large enough, such that at a given $x \in S_{j}$ the components of the perturbations due to the rest of the points of the set $S_{j}$ through the directions defined by the distribution $D$ do not destroy the transversality obtained at $B_{g_{k}}(x, c)$. The condition we impose to $\mathcal{N}$ is

$$
c^{\prime \prime} \delta\left(\log \left(\delta^{-1}\right)\right)^{-p} \geq 2 c_{u} c^{\prime} \delta \exp \left(-\lambda \mathcal{N}^{2}\right),
$$

where $c_{u}$ is a uniform constant. The left hand side of the inequality is the amount of transversality obtained by the perturbation at a point $x \in S_{j}$. The right hand side is a value greater than the double of the value of the norm of the sum of all the other perturbations in the set $S_{j}$ (different from the selected and multiplied by the uniform constant provided by the local and $C^{r}$-open property). So the inequality assures us that, after adding the perturbation, we have obtained $\mathcal{P}\left(\frac{1}{2} c^{\prime \prime} \delta\left(\log \left(\delta^{-1}\right)\right)^{-p}, y\right)$ at a c-neighborhood of $S_{j}$.

Now the process is clear. We perturb in $S_{1}$ to obtain $\mathcal{P}\left(\eta_{1}, x\right)$ at a $c$-neighborhood of it. Afterwards we add again a perturbation with mixed $C^{r}$-bounds $\left(\frac{1}{c_{u}} \eta_{1} / 2, c_{R}^{2}\right)$ to achieve $\mathcal{P}\left(\min \left(\eta_{2}, \eta_{1} / 2\right), y\right)$ for all $y$ at a $c$-neighborhood of $S_{1} \bigcup S_{2}$. The number of steps is independent of $k$, so the achieved final property is $\mathcal{P}(\eta, x)$ where $\eta$ does not depend on $k$. The added section has also mixed $C^{r}$-bounds $\left(\epsilon, c_{R}\right)$. The constant $\epsilon$ is obtained by adjusting the $\eta_{i}$ in the iteration, which is possible by property 1 . The constant $c_{R}$ is uniform because at each stage does depend only on the precedent values of $c_{R}$ and $\eta_{j}$, so it is independent of $k$, and thus the uniformity is obvious since the number of stages is independent of $k$ (i.e. $O\left(\mathcal{N}^{2 n+1}\right)$ ). Remark that $c_{R}$ cannot be bounded since property 1 provides uniformity but not control of the constant!

To end we have to check the inequality (4.1) in all the steps of the process. Following the asymptotic analysis of [Do96] we conclude that it is possible to obtain an integer $\mathcal{N}$ independently of the step $j$. This ends the proof.

4.2. The local perturbation. We are going to achieve the transversality property for $\partial F$, required in Theorem 2.10, by using Proposition 4.2. The first observation is the following

LEMMA 4.3. There exists a uniform constant $\xi>0$, such that, for $k$ large enough, any point $x$ verifying $|\partial F(x)|<\xi$ lies in the set $\Omega_{2 \xi}$.

The proof is identical to that of Lemma 3.1 and we refer again to [Do99] for details.

We are going to perturb the sequence of sections $s_{k}^{1}$ on order to obtain transversality. The open mixed $C^{2}$-open property $\mathcal{P}(\epsilon, x)$ which we have to obtain in $C$ is that $\partial F$ is $\epsilon$-transverse to $\mathbf{0}$ at $x$. We are in the hypothesis of Definition 4.1. Now we are going to construct a local section satisfying the hypothesis of Proposition 4.2, thus concluding the proof. We can choose $c>0$ uniformly to assure that $B_{g_{k}}(x, 8 c) \subset \Omega_{\xi}$ for any $x \in \Omega_{\frac{3}{2} \xi}$ and also that $B_{g_{k}}(x, 8 c)$ is in the complementary set of $\Omega_{\xi}$ for any $x$ in the complementary set of $\Omega_{\frac{3}{2}} \xi$.

If we are in the complementary set of $\Omega_{\frac{3}{2} \xi}$ we can choose the section $\tau_{k, x}$ to be zero, because of Lemma 4.3 . 
Choose a point $x \in \Omega_{\frac{3}{2} \xi}$. Take an approximately contact holomorphic chart $\left(s, z_{k}^{1}, \ldots, z_{k}^{n}\right)$ satisfying the properties of Corollary 2.4. Generalizing [Au00, MPS99] we define the 1 -forms

$$
\mu_{k}^{j}=\partial\left(\frac{z_{k}^{j} s_{k, x}^{r e f}}{s_{k}^{0}}\right)
$$

In $\Omega_{\xi}$ these forms have uniform mixed $C^{3}$-bounds because the term $\nabla^{r} \bar{\partial} z_{k}^{j}$ can be bounded near $x$, and furthermore both $s_{k, x}^{r e f}$ and $s_{k}^{0}$ have mixed $C^{3}$-bounds, as well as $s_{k}^{0}$ is also bounded below. Recall that $\left(\mu_{k}^{j}\right)_{j=1, \ldots, n}$ is a unitary basis of $D^{*}$ at $x$, and it is almost unitary in the ball $B_{g_{k}}(x, 8 c)$ (i.e. the basis $\mu_{k}^{j}$ is arbitrarily close to a unitary basis), after eventually shrinking $c$ uniformly. We define a map $v: B_{g_{k}}(x, 8 c) \rightarrow \mathbb{C}^{n}$ by the formula:

$$
\partial F=\sum_{j=1}^{n} v_{j} \mu_{k}^{j}
$$

In matrix notation,

$$
\partial F=v^{T} \cdot \mu_{k}
$$

where $\mu_{k}^{T}=\left(\mu_{k}^{1}, \ldots, \mu_{k}^{n}\right)$. Thus it is possible to understand $\mu_{k}$ as a linear map $\mu_{k}(y): \mathbb{C}^{n} \rightarrow T_{y}^{*} M$. Multiplying by $\mu_{k}^{-1}$ in (4.2) we obtain

$$
v^{T}=\partial F \cdot \mu_{k}^{-1}
$$

This implies that $|v|=O(1)$, since $\mu_{k}$ is approximately unitary. We compute now the derivatives of $v$ using equation (4.2). To do this, recall that $\partial F$ has mixed $C^{2}$-bounds in the ball $B_{g_{k}}(x, 8 c)$ (because $F$ has mixed $C^{3}$-bounds). Also, in the same way, $\mu_{k}$ has mixed $C^{2}$-bounds. Differentiating (4.2)

$$
\nabla \partial F=\nabla v^{T} \cdot \mu_{k}+v^{T} \cdot \nabla \mu_{k}
$$

which implies that $\left|\nabla v^{T} \cdot \mu_{k}\right|=O(1)$ and using again that $\mu_{k}$ is approximately unitary, we finally get

$$
|\nabla v|=O(1)
$$

Differentiating respect to $\bar{\partial}$ we find that $|\bar{\partial} v|=O\left(k^{-1 / 2}\right)$, and iterating the process

$$
|\nabla \nabla v|=O(1), \quad|\nabla \bar{\partial} v|=O\left(k^{-1 / 2}\right) .
$$

Now we use the approximately contact-holomorphic chart $\Psi$ defined in Corollary 2.4, after eventual uniform shrinking of $c$. We construct the function $\hat{v}=v \circ \Psi^{-1}$. By the properties of $\Psi$, it is easy to check that:

$$
|\hat{v}|=O(1), \quad\left|\nabla^{r} \hat{v}\right|=O(1), \quad\left|\nabla^{r-1} \bar{\partial} \hat{v}\right|=O\left(k^{-1 / 2}\right), \quad r=1,2 .
$$

Scaling the coordinates by a uniform constant we can assume that $\Psi\left(B_{g_{k}}(x, 2 c)\right) \subset$ $B_{2 n+1}(0,2) \subset \Psi\left(B_{g_{k}}(x, 8 c)\right)$. Now, we are in the hypothesis of the following

Proposition 4.4. Let $f_{k}: B \times[0,1] \rightarrow \mathbb{C}^{m}$ be a sequence of functions where $B$ is the ball of radius 1 in $\mathbb{C}^{n}$ and $B \times[0,1]$ is equipped with a sequence of contact forms 
$\theta(k)$ whose distributions are asymptotically flat. Let $0<\delta<1 / 2$ be a constant and let $\sigma=\delta\left(\log \left(\delta^{-1}\right)\right)^{-p}$, where $p$ is a integer depending only on the dimensions. Assume that $f_{k}$ satisfies over $B \times[0,1]$ the following bounds

$$
\left|f_{k}\right| \leq 1, \quad\left|\bar{\partial}_{0} f_{k}\right| \leq \sigma, \quad\left|\nabla \bar{\partial}_{0} f_{k}\right| \leq \sigma
$$

for $k$ large enough, where $\bar{\partial}_{0}$ is the $(0,1)$ operator defined in $D(k)=\operatorname{ker} \theta(k)$ by vertical projection of the standard complex structure $J_{0}$. Then for $k$ large enough there exists a smooth curve $w_{k}:[0,1] \rightarrow \mathbb{C}^{m}$ such that $\left|w_{k}\right|<\delta$ and the function $f_{k}-w_{k}$ is $\sigma$ transverse to zero on $B(0,1 / 2) \times[0,1]$. Moreover, if $\left|\partial f_{k} / \partial s\right|<1$ and $\left|\partial \nabla f_{k} / \partial s\right|<1$, we can choose $w_{k}$ such that $\left|d^{i} w_{k} / d s^{i}\right|<\Phi(\delta),(i=1,2) ; d^{j} w_{k} / d s^{j}(0)=0$ and $d^{j} w_{k} / d s^{j}(1)=0$, for all $j \in \mathbb{N}$, where $c$ is a uniform constant and $\Phi: \mathbb{R}^{+} \rightarrow \mathbb{R}^{+}$is a function depending only on the dimensions.

This proposition will be proved in Section 5. This is the analogous in the contact case to Theorem 12 in [Do99]. For the particular value of $m=1$ it has been proved in [IMP99].

Now we apply this proposition to the map $\hat{v}$ over $[-1,1] \times B$, because for $k$ large enough, it satisfies the hypothesis (without loss of generality we can choose $[-1,1] \times B$ instead of $[0,1] \times B$, also we suppose $|\hat{v}|_{C^{2}} \leq 1$, multiplying by uniform constants). The obtained path $\hat{w}$ is extended to $\mathbb{R} \times \mathbb{C}^{n}$ as

$$
\hat{w}(s, z)= \begin{cases}\hat{w}(1) & , \text { for } s>1 \\ \hat{w}(s) & , \text { for } s \in[-1,1] \\ \hat{w}(-1) & , \text { for } s<-1\end{cases}
$$

We keep the same notation for this extended map. The map $\hat{v}-\hat{w}$ is transverse to 0 in $B(0,1) \times[-1,1]$, thus $\hat{v}-\hat{w}$ is also transverse to 0 in $B_{2 n+1}(0,1)$. Remark that the chart $\Psi$ is defined in a ball of radius $O\left(k^{1 / 2}\right)$. So the pull-back of $\hat{w}$ by $\Psi$, denoted $w$, is well defined in this ball, and it will be enough for our purposes. By the properties of $\Psi$ we have that $v-w$ is $\eta$-transverse to 0 over the ball $B_{g_{k}}(x, c)$. The constant $\eta$ does not coincide with the transversality obtained by $\hat{v}-\hat{w}$ by a uniform factor, so $\eta=c_{u} \delta\left(\log \left(\delta^{-1}\right)\right)^{-p}$, the constants $c_{u}$ and $p$ are uniform and $\delta$ is the norm of $w$ up to a uniform factor $c_{u^{\prime}}$, i.e. $|w|<c_{u^{\prime}} \delta, c_{u^{\prime}} \neq 0$.

The needed perturbation is

$$
\tau_{k, x}=\sum_{j=1}^{n} w_{j} z_{j} s_{k, x}^{r e f}
$$

The first question is whether $\tau_{k, x}$ has the desired mixed $C^{3}$-bounds. The verification is mere routine and it follows the lines of [IMP99]. As an example, we compute the bound for $\bar{\partial} \tau_{k, x}$ :

$$
\bar{\partial} \tau_{k, x}=\sum \bar{\partial} w_{j} z_{j} s_{k, x}^{r e f}+w_{j} \bar{\partial} z_{j} s_{k, x}^{r e f}+w_{j} z_{j} \bar{\partial} s_{k, x}^{r e f} .
$$

The third term is easily bounded by

$$
\begin{aligned}
\left|w_{j} z_{j} \bar{\partial} s_{k, x}^{r e f}\right| & \leq O(1) 2 d_{k}(x, y) c_{a} k^{-1 / 2} P\left(d_{k}(x, y)\right) \exp \left(-\lambda d_{k}(x, y)^{2}\right)= \\
& =k^{-1 / 2} Q\left(d_{k}(x, y)\right) \exp \left(-\lambda d_{k}(x, y)^{2}\right) .
\end{aligned}
$$


The second one is bounded in the same way recalling that $\bar{\partial} z=c_{0} k^{-1 / 2} d_{k}(x, y)$, where $c_{0}>0$ is uniform. For the first term we proceed as follows:

$$
\left|\bar{\partial} w_{j} z_{j} s_{k, x}^{r e f}\right| \leq\left|\bar{\partial} w_{j}\right| P\left(d_{k}(x, y)\right) \exp \left(-\lambda d_{k}(x, y)^{2}\right) .
$$

Recall that $\theta_{k^{1 / 2}}$ is asymptotically flat. Using this fact we obtain that $\left|\nabla w_{j}\right| \leq$ $O\left(k^{-1 / 2}\right) d_{k}(x, y)$, thus in particular $\left|\bar{\partial} w_{j}\right| \leq O\left(k^{-1 / 2}\right) d_{k}(x, y)$. This concludes the bounding.

Now we have to compare $\tau_{k, x}$ with $w$. If $\partial \frac{\tau_{k, x}}{s_{k}^{0}}$ were $w^{T} \mu_{k}$, the proof would be rapidly concluded. But we are almost in this situation because

$$
\partial \frac{\tau_{k, x}}{s_{k}^{0}}=\Sigma \partial w_{j} \frac{z_{j} s_{k, x}^{r e f}}{s_{k}^{0}}+\Sigma w_{j} \mu_{j} .
$$

Using (3.2) again, we obtain

$$
\partial \frac{\tau_{k, x}}{s_{k}^{0}}=O\left(k^{-1 / 2}\right)+w^{T} \mu_{k}
$$

Therefore, we can find $\tilde{w}$ such that $\partial \frac{\tau_{k, x}}{s_{k}^{0}}=\tilde{w}^{T} \mu_{k}$ and $|w-\tilde{w}|=0\left(k^{-1 / 2}\right)$. So, for $k$ large enough $v-\tilde{w}$ is, say, $0.9 \eta$-transverse to 0 in $B_{g_{k}}(x, c)$. We end by remarking that $\partial \frac{s_{k}^{1}-\tau_{k, x}}{s_{k}^{0}}$ is $c_{b} \eta$-transverse to $0, c_{b}>0$ a uniform constant, if $v-\tilde{w}$ is $0.9 \eta$-transverse to $\mathbf{0}$. This is obvious since both just differ by the application of the almost unitary matrix $\mu_{k}$.

5. Local results. The aim of this section is to prove Theorem 4.4. This is the generalization of the local results in the symplectic setting, needed to achieve controlled transversality in the contact case.

5.1. Reduction to integral distributions. We can easily reduce the proof to the following

Proposition 5.1. Let $f: B \times[0,1] \rightarrow \mathbb{C}^{m}$ be a complex valued function, where $B$ is the ball of radius 1 in $\mathbb{C}^{n}$. Let $0<\delta<1 / 2$ be a constant and let $\sigma=\delta\left(\log \left(\delta^{-1}\right)\right)^{-p}$, where $p$ is a suitable fixed integer depending only on the dimensions $n, m$. Assume that $f_{s}$ satisfies the following bounds over $B \times[0,1]$

$$
\left|f_{s}\right| \leq 1, \quad\left|\bar{\partial} f_{s}\right| \leq \sigma, \quad\left|\nabla \bar{\partial} f_{s}\right| \leq \sigma .
$$

Then there exists a smooth curve $w:[0,1] \rightarrow \mathbb{C}^{m}$ such that $|w|<\delta$ and the function $f_{s}-w(s)$ is $\sigma$-transverse to zero over the ball $B(0,1 / 2)$. Moreover, if $\left|\partial f_{s} / \partial s\right|<1$ and $\left|\partial \nabla f_{s} / \partial s\right|<1$, we can choose $w$ such that $\left|d^{i} w / d s^{i}\right|<\Phi(\delta)(i=1,2), d^{j} w / d s^{j}(0)=0$ and $d^{j} w / d s^{j}(1)=0$ for all $j \in \mathbb{N}$, where $\Phi$ is a function depending only on the dimensions $n, m$.

The proof of this Proposition is a generalization of Lemma 10 in [IMP99] to the case $m>1$.

Proof of Theorem 4.4. We have to obtain the transversality of $f_{k}-w_{k}$ when we restrict $\nabla\left(f_{k}-w_{k}\right)$ to the distribution defined by $\theta(k)$. Recall that $\theta(k)$ is asymptotically flat, so $\angle_{M}\left(D_{h}, D_{k}\right)=O\left(k^{-1 / 2}\right)$, where $D_{h}=\mathbb{C}^{n} \times\{p\}$ and $D_{k}=\operatorname{Ker} \theta(k)$. The key idea is that $\nabla\left(f_{k}-w_{k}\right)=O(1)$, so if $f_{k}-w_{k}$ is $\eta$-transverse to $D_{h}$, then it is, say, $0.9 \eta$-transverse to $D_{k}$ for $k$ large enough. The factor 0.9 can be eliminated by increasing $p$ uniformly. 
5.2. Proof of Proposition 5.1. Let $f: \mathbb{C}^{n} \rightarrow \mathbb{C}^{m}$ be a smooth map. We define the subset $U(f, w, \delta, \sigma)$ of the ball $B(w, \delta)$ of radius $\delta$ as the set of points where $f$ is $\sigma$-transverse to $w$ all over the ball $\frac{1}{2} B^{2 n}$, the ball of radius $1 / 2$ in $\mathbb{C}^{n}$. First we prove the following

TheOREM 5.2 (Extension of Theorem 12 in [Do99]). For any $n, m, \delta>0$ and $0<\gamma<1$ there is a $p=p(n, m, \gamma)$ such that if we define $\sigma=\delta\left(\log \left(\delta^{-1}\right)\right)^{-p}$ then for all the maps $f: B^{2 n} \rightarrow \mathbb{C}^{m}$ verifying that

$$
|f| \leq 1, \quad|\bar{\partial} f| \leq \sigma, \quad|\nabla \bar{\partial} f| \leq \sigma,
$$

there exists a connected component of $U(f, w, \delta, \sigma)$ containing another path-connected set $U^{\prime}(f, w, \delta, \sigma)$ whose volume is at least $\gamma$ times the total volume of $B(w, \delta)$ and such that given two points $x, y \in U^{\prime}(f, w, \delta, \sigma)$ is possible to find a smooth curve $\gamma$ in $U(f, w, \delta, \sigma)$ joining $x$ and $y$ with curvature at each point and length bounded by $\Phi(\delta)$, where $\Phi$ is a function depending only on the dimensions $n, m$.

We repeat the proof of Theorem 12 of [Do99] taking care of some details.

Proof. The first step in the proof is to approximate $f_{s}$ by a holomorphic function $\hat{f}_{s}$ such that $\left|f_{s}-\hat{f}_{s}\right|_{B, C^{1}}<c \sigma$ (see Lemma 28 in [Do96]). This process does not hold over all of the unit ball. This is the reason why we restrict ourselves to the ball $B^{\prime}=\frac{1}{2} B^{2 n}$ in the sequel. Then we approximate $\hat{f}_{s}$ by a polynomial. We can obtain polynomials $g_{s}$ such that $\left|g_{s}-f_{s}\right|_{B^{\prime}, C^{1}} \leq c \sigma$ and their degree $d$ can be estimated by $O\left(\log \left(\sigma^{-1}\right)\right)$.

Adapting notations of [Au97, IMP99] we denote by $Z_{h_{s}, \epsilon}$ the images of the set of points of $B^{\prime}$ which are not $\epsilon$-transverse to 0 for $h_{s}$. We want to prove that one component of the complementary set of $Z_{f_{s}, \sigma}$ satisfies the required properties. The first observation is that the $C^{1}$-closedness of $f_{s}$ and $g_{s}$ assures us that $Z_{f_{s}, \sigma} \subset Z_{g_{s},(c+1) \sigma}$.

We use now the following

THEOREM 5.3 (Theorem 26 of [Do99]). Given a polynomial $g: \mathbb{C}^{n} \rightarrow \mathbb{C}^{m}$ of degree $d$ and $\epsilon>0$ there is a real-algebraic subvariety $A(g) \subset \mathbb{C}^{m}$ of codimension 2 and degree $D$ such that $Z_{g, \epsilon}$ is contained in the $K \epsilon$-neighborhood of $A(g)$, where $K, D \leq(d+1)^{p}$, for some integer $p$ depending only on the dimensions $n, m$.

Given a hypersurface $A$ define $A_{\epsilon}$ to be the $\epsilon$-neighborhood of $A$. We use the following Donaldson's result:

Proposition 5.4 (Proposition 31 in [Do99]). For each integer $N$ and real number $\theta>0$, there is a $\mu=\mu(\theta, N)$ with the following property. For any real-algebraic hypersurface $A \subset \mathbb{R}^{n}$ of degree $D$ and $\epsilon \leq(D+1)^{\mu}$,

$$
\operatorname{Vol}\left(B^{N} \cap A_{\epsilon}\right) \leq \theta \text {. }
$$

Denote $\sigma^{\prime}=(c+1) \sigma$. With these two results and following the discussion in [Do96] p. 689 about the behavior of the function $\delta\left(\log \delta^{-1}\right)^{-p}$ we can assure that the $\sigma^{\prime}=\delta \log \left(\delta^{-1}\right)^{-p}$-neighborhood, $p$ a fixed integer, of the bad set $A\left(g_{s}\right)$ has volume arbitrarily small. Also we can assure the same condition for the $3 \sigma^{\prime}$-neighborhood (changing $p$ slightly). We take a covering of $B(0, \delta)$ by balls $B\left(x_{i}, \sigma^{\prime} / 2\right)$ of centers $x_{i}$ and radius $\sigma^{\prime} / 2$ and assuring that the covering of balls with radius $\sigma^{\prime}$ centered in the same points cover each point of $B(0, \delta)$ only a finite uniform number of times, for instance less than $\nu$ times. Denote by $\mathcal{C}$ the set of centers $x_{i}$ of the balls of the 
covering contained in the $2 \sigma^{\prime}$-neighborhood of $A\left(g_{s}\right)$. Recall that the union of these balls is contained in $A_{3 \sigma^{\prime}}\left(g_{s}\right)$, so we can conclude that

$$
\sum_{x_{i} \in \mathcal{C}} \operatorname{vol}\left(B\left(x_{i}, \sigma^{\prime}\right)\right) \leq \nu \operatorname{vol}\left(A_{3 \sigma^{\prime}}\left(g_{s}\right)\right) .
$$

And from this expression we can easily obtain a bound for the number of points in $\mathcal{C}$ as

$$
\operatorname{card}(\mathcal{C}) \leq c_{u} \delta^{2 n} /\left(\sigma^{\prime}\right)^{2 n}
$$

where $c_{u}$ is a uniform constant and so this number only depends on $\delta$. But observe that

$$
A_{\sigma^{\prime}}\left(g_{s}\right) \subset \bigcup_{x_{i} \in \mathcal{C}} B\left(x_{i}, \sigma^{\prime} / 2\right) \subset \bigcup_{x_{i} \in \mathcal{C}} B\left(x_{i}, \sigma^{\prime}\right)=W_{s} \subset A_{3 \sigma^{\prime}}\left(g_{s}\right)
$$

Now following the proof of Theorem 12 in [Do99] (that we do not detail) we find that there is a connected component $U^{\prime \prime}$ of the complementary of $A_{\sigma^{\prime}}\left(g_{s}\right)$ which volume arbitrarily close to the total one. Obviously, there must be connected components $U^{\prime}$ and $U$ of the complementaries of $W_{s}$ and $A_{3 \sigma^{\prime}}\left(g_{s}\right)$ satisfying $U^{\prime \prime} \subset U^{\prime} \subset U$. We will assume that $U^{\prime}=U^{\prime}(f, w, \delta, \sigma)$ and $U=U(f, w, \delta, \sigma)$ are the sets in the statement of the Theorem. To finish we have only to check that these two sets satisfy the required properties.

We adapt the ideas of [IMP99]. We call $N=\operatorname{card}(\mathcal{C})$. Observe that we have fixed $\sigma$, and then $N=f(\delta)$ and $2 \pi \sigma N=g(\delta)$, for some functions $f$ and $g$. Now take $y, z$ points in the large connected component $U$ of the complementary of $Z_{s}^{+}$. Denote $L(y, z)$ the straight segment joining them. This segment cuts at most at $2 N$ points $y_{0}, z_{0}, y_{1}, \ldots$ to the border Bor $=\partial\left(\bigcup_{x_{i} \in \mathcal{C}} B\left(x_{i}, \sigma^{\prime}\right)\right)$. Obviously $L\left(z_{i}, y_{i+1}\right) \subset U$ and $L\left(y_{i}, z_{i}\right) \subset \bigcup_{x_{i} \in \mathcal{C}} B\left(x_{i}, \sigma^{\prime}\right)$. We replace the lines $L\left(y_{i}, z_{i}\right)$ by curves $C\left(y_{i}, z_{i}\right)$ contained in Bor connecting $y_{i}$ and $z_{i}$. We construct the curves following maximal diameters of the spheres which define the border and so length $\left(C\left(y_{i}, z_{i}\right)\right) \leq g(\delta)$. Therefore the curve

$$
\gamma^{\prime}=L\left(y, y_{0}\right) \bigcup C\left(y_{0}, z_{0}\right) \bigcup L\left(z_{0}, y_{1}\right) \cdots
$$

satisfies

$$
\begin{aligned}
\text { length }\left(\gamma^{\prime}\right)= & L\left(y, y_{0}\right)+L\left(z_{q}, z\right)+\Sigma L\left(y_{i}, y_{i+1}\right)+\Sigma C\left(y_{i}, z_{i}\right) \leq 2 \delta+ \\
& +f(\delta) g(\delta)=\Phi(\delta) / 2
\end{aligned}
$$

where $\Phi$ is some function depending only on the dimensions. Perturbing slightly $\gamma^{\prime}$ to make it differentiable and removing it from the border we obtain $\gamma$ which, bounding enough the perturbation, satisfies length $(\gamma) \leq \Phi(\delta)$. So the length between two points can be bounded by a function of $\delta$. Moreover, if we translate the diameters in the border $B o r$ till the border of $\bigcup_{x_{i} \in \mathcal{C}} B\left(x_{i}, \sigma^{\prime} / 2\right)$, we can assure that the curvature of the path can be bounded by $O\left(1 / \sigma^{\prime}\right)$, again a function of $\delta$.

With the precedent result we can easily finish the proof of Proposition 5.1. Recall that $f_{s}$ satisfies

$$
\left|\frac{\partial f_{s}}{\partial s}\right| \leq 1, \quad\left|\frac{\partial \nabla f_{s}}{\partial s}\right| \leq 1
$$


This implies by a simple application of the Mean Value Theorem of the Differential Calculus (for vectorial maps) that:

$$
\left|f_{s}-f_{s+\epsilon}\right|<\epsilon, \quad\left|\nabla f_{s}-\nabla f_{s+\epsilon}\right|<\epsilon .
$$

Recall that given two maps $f, g: \mathbb{C}^{n} \rightarrow \mathbb{C}^{m}$ such that $|f-g|_{C^{1}}<\epsilon$, if $f$ has a left inverse with norm less than $\eta^{-1}$, for a large enough uniform constant $c_{0}, g$ has a left inverse of norm less than $\left(\eta-c_{0} \epsilon\right)^{-1}$. This implies that

$$
U\left(f_{s}, 0, \delta, \sigma\right) \subset U\left(f_{s+\epsilon}, 0, \delta, \sigma-c_{0} \epsilon\right)
$$

We choose a number $\sigma$, which satisfies the hypothesis of Theorem 5.2, with $\gamma>\frac{1}{2}$. Take an integer $q$ satisfying that

$$
\frac{1}{q}<\sigma / c_{0}
$$

We can choose $x_{i} \in U\left(g_{i / q}, 0, \delta,(c+1) \sigma\right) \bigcap U\left(g_{(i+1) / q}, 0, \delta,(c+1) \sigma\right)$, for $i=0, \ldots, q-1$. Consider the path $H_{i}=x_{i} \times[i / q,(i+1) / q]$. There exists a smooth path $V_{i}$ connecting $x_{i}$ with $x_{i+1}$ in $U^{\prime}\left(g_{(i+1) / q}, 0, \delta, \sigma\right)$. Its length is bounded above by $\Phi(\delta)$, as well as its curvature at any point. We parametrize $V_{i}$ by its arch-length.

We choose a smooth function $\beta:[0,1] \rightarrow[0,1]$ satisfying:

$$
\beta(x)= \begin{cases}0, & x \in[0,1 / 4] \\ 0<\beta(x)<1, & 1 / 4<x<3 / 4 \\ 1, & x \in[3 / 4,1]\end{cases}
$$

and compute $|\beta|_{C_{2}}=c_{b}$. Denote $\beta_{i}(x)=\beta(x) \cdot \operatorname{length}\left(V_{i}\right)$. This function has norm $\left|\beta_{i}\right|_{C_{2}}<c_{b} \Phi(\delta)$. Define a path

$$
w\left((i / q+\epsilon)=V_{i}\left(\beta_{i}\left(q\left(\epsilon+\frac{1}{2 q}\right)\right)\right), \quad|\epsilon| \leq \frac{1}{2 q} .\right.
$$

The map $w$ is smooth and we obtain $|w|_{C_{2}}<c_{b} \Phi(\delta)$, a bound which is a function of $\delta$. Remark that the first derivative depends on the length of the path $V_{i}$ and the second on its curvature. Finally we observe that by (5.1) and (5.2) $w(s)$ is $\sigma / 2$ transverse to $\mathbf{0}$, for all $s \in[0,1]$. We can find an integer $p^{\prime}$ such that $\sigma^{\prime}=\delta\left(\log \left(\delta^{-1}\right)\right)^{-p^{\prime}}<\sigma / 2$ and the proof is finished.

6. Topological considerations. In this section we will do some simple topological remarks about the contact Lefschetz pencils. In the first subsection we will study the topological relationship between the smooth fibers of a contact fibration. In the second one we study the relationship from a contact geometry point of view.

We fix $\left(\mathbb{R}^{2 n}, \omega_{0}\right)$ with the standard symplectic form $\omega_{0}=\Sigma d x_{i} \wedge d y_{i}$. Choose an integer $0 \leq k \leq n$ and define the Morse function $f=-x_{1}^{2} \cdots-x_{k}^{2}+x_{k+1}^{2} \cdots+y_{n}^{2}$. The set $f^{-1}(-\epsilon, \epsilon)$ is a $k$-dimensional handle. The descendent sphere $S_{-\epsilon}=\left\{x_{k+1}=\right.$ $\left.\cdots=y_{n}=0, x_{1}^{2}+\cdots+x_{k}^{2}=\epsilon\right\}$ is an isotropic submanifold. The level set $f^{-1}(-\epsilon)$ is a contact manifold. It can be shown that any isotropic sphere $S$ inside a contact manifold $C$ admits a trivialization changing the picture to the model given by $S_{-\epsilon}$ inside $f^{-1}(\epsilon)$. So we can perform a surgery using the local model obtaining a new contact manifold. This is usually called an isotropic surgery of index $k$ [We91]. In the particular case where $k=n$ we will say that the surgery is Legendrian, because the descendent sphere is Legendrian. 
The result that we will prove in the two following subsections is

Proposition 6.1. Given a contact fibration $(f, \Delta, A)$, any pair of regular fibers are related, for $k$ large enough, by a sequence of legendrian Weinstein surgeries.

6.1. Crossing critical curves. Over each of the connected components of $\mathbb{C P}^{1}-f_{k}(\Delta)$ the fibers of $f_{k}$ are isotopic. Now, we are interested in analyzing the topological behavior of the fibers when crossing through $\Delta$. We will prove in this subsection the following

Proposition 6.2. Given a contact fibration $(f, \Delta, A)$, choose a path $\gamma:[0,1] \rightarrow$ $\mathbb{C P}^{1}$ such that $\gamma(1 / 2) \in f(\Delta)$ and the rest of the points of $\gamma$ are regular values of $f$. Then $N=f^{-1}(\gamma(1))$ is built up from $N^{\prime}=f^{-1}(\gamma(0))$ by adding a $n$-dimensional handle and removing another $n$-dimensional handle. Therefore $H_{j}(N)=H_{j}\left(N^{\prime}\right)$ (resp. $\pi_{j}(N)=\pi_{j}\left(N^{\prime}\right)$ ) for $j=0, \ldots, n-2$.

This proves the first part of Proposition 6.1.

Proof. We restrict ourselves without loss of generality to neighborhoods of $\gamma(1 / 2)$ and of the critical point of $f$, where we can define a compatible chart. So, with the usual identifications, we can suppose that $f: \mathbb{R} \times \mathbb{C}^{n} \rightarrow \mathbb{C}$. Moreover, for simplicity, we will assume that $f\left(s, z_{1}, \ldots, z_{n}\right)=s+z_{1}^{2}+\cdots+z_{n}^{2}$ (being the general case a straightforward generalization) and the path will be $\gamma(t)=2(t-1 / 2) i$ with a critical value for $f$ at $t=1 / 2$.

The proof of the result reduces to show that $B=f^{-1}(\gamma([0,1]))$ is a cobordism between $N=f^{-1}(\gamma(0))$ and $N^{\prime}=f^{-1}(\gamma(1))$ with only one surgery of index $n$. And this follows if we find a Morse function with a critical point of index $n$. Choose $h=i m(f)=2 x_{1} y_{1}+2 x_{2} y_{2}+\cdots+2 x_{n} y_{n}$. We can assume at a neighborhood $U$ of the critical point $\mathbf{0}=(0, \ldots, 0)$ that $B \bigcap U \subset g^{-1}(0)$, where $g\left(s, x_{1}, \ldots\right)=\operatorname{Re}(f)=$ $s+x_{1}^{2}+y_{1}^{2}+\ldots+x_{n}^{2}+y_{n}^{2}$. To compute the index of $g$ we have to restrict ourselves to $\operatorname{ker} \nabla g(\mathbf{0})$ which is

$$
\operatorname{ker} \nabla g(\mathbf{0})=\left\{\left(0, z_{1}, \ldots, z_{n}\right):\left(z_{1}, \ldots, z_{n}\right) \in \mathbb{C}^{n} .\right\}
$$

Finally, recall that

$$
\nabla \nabla h=\left(\begin{array}{ccc}
0 & 0 & 0 \\
0 & 0 & 2 I_{n} \\
0 & 2 I_{n} & 0
\end{array}\right)
$$

Restricting to $\operatorname{ker} \nabla g(\mathbf{0})$ we obtain

$$
\nabla \nabla h_{\mid \operatorname{ker} \nabla g(0)}=\left(\begin{array}{cc}
0 & 2 I_{n} \\
2 I_{n} & 0
\end{array}\right),
$$

which has index $n$.

Now we can obtain a geometrical relationship between the contact submanifolds obtained in Theorem 2.9 as zero sets of transverse sequences with mixed $C^{3}$-bounds. The result will be

CoROllaRY 6.3. Let $S$ be a line bundle with connection over a closed exact contact manifold $(C, D)$ Given $N_{k}$ and $N_{k}^{\prime}$ sequences of contact submanifolds obtained as zero sets of transverse sections with mixed $C^{3}$-bounds of the bundles $S \otimes L^{\otimes k}$, then $N_{k}$ and $N_{k}^{\prime}$ are cobordant through a cobordism defined by surgeries of index $n$. 
Proof. Initially we will suppose that the almost complex structure $J$ used in the definition and construction of the sequences of sections are coincident in the two cases.

Choose two of such sequences $\sigma_{k}^{1}$ and $\sigma_{k}^{2}$ which are $\eta$-transverse to $\mathbf{0}$, for some $\eta>0$. We claim that we can assume without loss of generality that $\sigma_{k}^{1} \oplus \sigma_{k}^{2}$ is $\eta^{\prime}$ transverse to $\mathbf{0}$, for some $\eta>0$. If we suppose this to be false, we can apply Theorem 2.9 and find a sequence of sections $\tau_{k}^{1} \oplus \tau_{k}^{2}$ with mixed $C^{3}$-bounds $\left(\eta / 2, c_{R}\right)$, such that $\left(\sigma_{k}^{1}+\tau_{k}^{1}\right) \oplus\left(\sigma_{k}^{2}+\tau_{k}^{2}\right)$ are $\eta^{\prime}$-transverse to $\mathbf{0}$. Define

$$
\sigma_{k, t}^{j}=\sigma_{k}^{j}+t \tau_{k}^{j}, \quad j=1,2
$$

It is easy to check that the sections $\sigma_{k}^{j}(t)$ are $\eta / 2$-transverse to $\mathbf{0}$, therefore its zero sets are isotopic. So we can impose directly that $\sigma_{k}^{0} \oplus \sigma_{k}^{1}$ is transverse to 0 .

Define the function

$$
\begin{aligned}
F_{k}: C-Z\left(\sigma_{k}^{1} \oplus \sigma_{k}^{2}\right) & \rightarrow \mathbb{C P}^{1} \\
p & \rightarrow \frac{\sigma_{k}^{2}(p)}{\sigma_{k}^{1}(p)} .
\end{aligned}
$$

By an analogous argument to that in the precedent lines we assume that $\partial F_{k}$ is $\eta$ transverse to 0 away from $Z\left(\sigma_{k}^{1} \oplus \sigma_{k}^{2}\right)$ Recall that $F_{k}^{-1}(0)=Z\left(\sigma_{k}^{1}\right)$ and $F_{k}^{-1}(\infty)=$ $Z\left(\sigma_{k}^{2}\right)$. Following the proof of Section 3 we obtain a contact fibration $f_{k}$, for $k$ large enough. But, we do not impose the local model at a neighborhood of $A$, therefore we do not perturb any neighborhood of $A$. We observe that the perturbation at a neighborhood of the set of critical points $\Delta$ does not change the counter-images of the points 0 and $\infty$. It follows since there exists a $\rho_{0}$-neighborhood, being $\rho_{0}>0$ a uniform constant, of these two fibers which does not intersect the "bad set" $\Gamma$, because of the mixed $C^{3}$-bounds of the sections $\sigma_{k}^{1}$ and $\sigma_{k}^{2}$ (which assure contactness at a neighborhood of the zero set of the section). So we perturb the sequence $F_{k}$ in a $\rho$-neighborhood of $\Delta$, with $0<\rho<\rho_{0}$. Therefore, even after performing the needed perturbations, $f_{k}^{-1}(0)=N_{k}$ and $f_{k}^{-1}(\infty)=N_{k}^{\prime}$.

By Proposition 6.2 we obtain, following a path between 0 and $\infty$, that $f_{k}^{-1}(0)=N_{k}$ and $f_{k}^{-1}(\infty)=N_{k}^{\prime}$ are related by a sequence of operations of index $n$. This finishes the proof if we admit that the complex structures coincide for the two sequences of sections.

Suppose now that the sequence of submanifolds $N_{k}$ is the zero set of a sequence of sections $\sigma_{k}$ with mixed $C^{3}$-bounds respect to an almost complex structure $J_{0}$. Furthermore $N_{k}^{\prime}$ comes from $s_{k}^{\prime}$ with mixed $C^{3}$-bounds respect to $J_{1}$. Fix a continuous path $J_{t}$ joining $J_{0}$ and $J_{1}$ in the moduli of compatible almost complex structures. We use the following

LEMmA 6.4. Let $J_{0}$ be a compatible almost complex structure in $C$. There exists a uniform $\epsilon>0$ satisfying that for any compatible almost complex structure $J$ such that $\left|J-J_{0}\right|<\epsilon$, there exist two sequences of sections $s_{k}$ and $s_{k}^{\prime}$ with mixed $C^{3}$-bounds, respect to $J_{0}$ and $J$, which are $\eta$-transverse to $\mathbf{0}$, for some $\eta>0$. Moreover the zero sets of $s_{k}$ and $s_{k}^{\prime}$ are isotopic for $k$ large enough.

Find the uniform constants $\epsilon_{t}=\epsilon$ provided by Lemma 6.4 for the almost complex structures $J_{t}$. By the continuity of $J_{t}$, there exists $\epsilon_{t}^{\prime}>0$ such that we can find two sequences of sections $s_{k}^{t}$ and $s_{k}^{t^{\prime}}$ with mixed $C^{3}$-bounds respect to $J_{t}$ and $J_{t+\epsilon_{t}^{\prime}}$ whose zero sets are isotopic, for $k$ large enough. 
Now we cover the segment $[0,1]$ by open segments $\left(t, t+\epsilon_{t}^{\prime}\right)$. The segment is compact, so we can find a finite subset $\left(0, \epsilon_{0}^{\prime}\right),\left(t_{1}, t_{1}+\epsilon_{t_{1}}^{\prime}\right), \ldots\left(t_{N}, 1\right)$ of the precedent set of segments which covers $[0,1]$. Obviously, without loss of generality, we can choose $t_{j}+\epsilon_{t_{j}}^{\prime}=t_{j+1}$.

Denote $Z(s)$ the zero set of a given section $s$. Observe that the sets $Z\left(\sigma_{k}\right)$ and $Z\left(s_{k}^{0}\right)$ are related through a cobordism of index $n$, for $k$ large enough. But, recall now that $Z\left(s_{k}^{0}\right)$ and $Z\left(s_{k}^{0^{\prime}}\right)$ are isotopic for $k$ large enough. Again, $Z\left(s_{k}^{0^{\prime}}\right)$ and $Z\left(s_{k}^{t_{1}}\right)$ are related through surgeries of index $n \ldots$ Following this argument we find that $Z\left(\sigma_{k}^{0}\right)$ and $Z\left(\sigma_{k}^{1}\right)$ are related through surgeries of index $n$.

REMARK 6.5. We cannot make the construction independent of the choice of contact form. For instance, if we change $\theta$ by $2 \theta$ the degree $2 k$ contact pencils associated to $\theta$ will be equivalent to the degree $k$ contact pencils associated to $2 \theta$. Obviously these families of pencils are not equivalent; moreover, they are related by a doubling degree operation like in the symplectic case.

What we could prove is that the doubling degree operation keeps the homology and homotopy groups till the needed order, because it is simply to construct a GromovGompf gluing of two copies of the submanifold along a codimension two submanifold inside them. (Not exactly the Gromov-Gompf symplectic sum, but the natural contact analogue) We do not give the details of this construction.

Proof of Lemma 6.4. Construct a sequence of sections $\sigma_{k} \eta$-transverse to 0 using the globalization argument of Proposition 4.2, for some uniform constant $\eta>0$. Thus, we obtain

$$
\sigma_{k}=\sum_{j \in J} w_{k} \cdot s_{k, x_{j}}^{r e f}
$$

where the points $x_{j}$ are elements of the set $S$ with the properties described in the proof of that Proposition. Recall that the definition of $\sigma_{k}$ makes sense if we change the almost complex structure $J_{0}$ by another one $J$. The obtained section $\sigma_{k}^{\prime}$ will be different because the reference sections $s_{k, x_{j}}^{r e f}$ depend on the complex structure. It is clear that $\sigma_{k}^{\prime}$ has mixed $C^{3}$-bounds with respect to $J$. We claim that $\sigma_{k}$ and $\sigma_{k}^{\prime}$ are $C^{1}$-close.

The steps to prove this are :

1. Check that $\left|s_{k, x, J_{0}}^{r e f}(y)-s_{k, x, J}^{r e f}(y)\right|_{C^{2}}=O\left(\epsilon \exp -d_{k}(x, y)^{2} / 4\right)$.

2. Check that the sum of all the reference sections still satisfies $\left|\sigma_{k}-\sigma_{k}^{\prime}\right|_{C^{2}}=$ $O(\epsilon)$.

Proposition 6.3 is weaker than the "contact Lefschetz hyperplane theorem" proved in [IMP99], but it is more geometrical and enlightens the behavior of the generic sections of the bundles $L^{\otimes k}$. It would be interesting to study the different connected components of $\mathbb{C P}^{1}-\Delta$ to control the topology of all the "approximately holomorphic" contact zeroes of $L^{\otimes k}$.

6.2. Contact information. In this subsection we will understand what happens with the contact structure of a fiber when we cross through a critical curve. The first thing to say is that fibers in the same connected component of $\mathbb{C P}^{1}-f(\Delta)$ are contactomorphic because of the Gray's stability theorem. 
The change in the contact structure when we cross a critical point is summarized in the following

Proposition 6.6. Given a contact fibration $(f, \Delta, A)$. Choose a path $\gamma:[0,1] \rightarrow$ $\mathbb{C P}^{1}$. Suppose that $\gamma$ only intersects $f(\Delta)$ at the point $\gamma(1 / 2)$. Denote by $v$ the unitary tangent vector defined in $\gamma(1 / 2)$ by the oriented curve $f(\Delta)$. Assume that $\left\{\frac{d \gamma(1 / 2)}{d t}, v\right\}$ is a positively oriented basis, then $f^{-1}(\gamma(0))$ and $f^{-1}(\gamma(1))$ are related through a symplectic cobordism with a Weinstein surgery along a Legendrian sphere in $f^{-1}(\gamma(0))$.

This proves the second part of Proposition 6.1.

Proof. Just copy all the setup of Proposition 6.2. The only change will be the assumption about $\phi$. We will assume here, without loss of generality, that $\phi(s)=i \cdot s$. Therefore the curve $\gamma$ is assumed to be laying on the real axis. The cobordism is simply $B=f^{-1}([0,1])$. There is a neighborhood $U$ of the critical point $p$ (which is the one in the fiber $f^{-1}(0)$ ) where the local model for $f$ holds. A Morse function for the cobordism in this case is $\operatorname{Re}(f)=h=x_{1}^{2}-y_{1}^{2}+\cdots+x_{n}^{2}-y_{n}^{2}$ which is defined on $B \bigcap U$. Therefore, where the local model holds, the cobordism is defined in $\mathbb{R} \times \mathbb{C}^{n}$ by the equation $s+2 x_{1} y_{1}+\cdots+x_{n} y_{n}$, which is a hypersurface. We can define a diffeomorphism

$$
\begin{aligned}
\phi: \mathbb{C}^{n} & \rightarrow \mathbb{R} \times \mathbb{C}^{n} \\
\left(z_{1}, \ldots, z_{n}\right) & \rightarrow\left(-2 x_{1} y_{1}-\cdots-2 x_{n} y_{n}, z_{1}, \ldots, z_{n}\right) .
\end{aligned}
$$

Recall that the form $d \theta$ induces at the critical value (the point $(0, \ldots, 0)$ in the trivialization) the standard pre-symplectic form $\omega_{0}=\sum d x_{j} \wedge d y_{j}$. Recall that for increasing $k \omega_{0}$ and $d \theta$ are closer and closer.

Check that $\phi^{*} \omega_{0}=\omega$ which is the standard symplectic form in $\mathbb{C}^{n}$. Now, through the diffeomorphism $\phi$, the fibers of $B$ (in a neighborhood of $U$ ) are just the level sets in $\mathbb{C}^{n}$ of the Morse function $\phi^{*} h=x_{1}^{2}-y_{1}^{2}+\cdots+x_{n}^{2}-y_{n}^{2}$. Fortunately the picture that we have got is exactly the local model defined in [We91] for the Legendrian gluing of a symplectic cobordism, when they are defined along a Legendrian $(n-1)$-sphere defined by the equation

$$
S_{E}(\epsilon)=\left\{\left(x_{1}, y_{1}, \ldots, y_{n}\right): x_{1}=\cdots=x_{n}=0, y_{1}^{2}+\cdots+y_{n}^{2}=\epsilon\right\} .
$$

This almost finishes the proof. Recall, now, that all the construction we have done is only approximated because we are using $\omega_{0}$ instead of $d \theta$. But, what we know is that $S_{E}(\epsilon)$ is totally real and asymptotically Legendrian when $k$ is large and $\epsilon$ is small enough (this second condition is needed to be inside $U$ ). We can bring back $S_{E}(\epsilon)$ to $f^{-1}(\gamma(0))$, denoting this new "approximately Legendrian" sphere by the same letter. To finish what we need to do is to change $f^{-1}\left(\gamma_{0}\right)$ by another very close contact manifold for which $S_{E}(\epsilon)$ is actually Legendrian. By Gray's stability theorem the proof is finished.

We have proved that all the fibers in a contact fibration are related through Legendrian surgeries. This could have important consequences. First at all, it could be possible to define invariants in 5 dimensional manifolds simply by choosing 3 dimensional invariants preserving Legendrian surgeries.

A first result in this direction is 
THEOREM 6.7. Given a symplectically fillable contact manifold, all its contact pencils have symplectically fillable fibers.

This is proved in [Pr01] and it is a corollary of the existence of pencils in symplectic manifolds with boundary.

REMARK 6.8. Giroux and Mohsen's paper [GM01], in particular, shows that the fibers of the contact pencils are always holomorphically fillable (a stronger result than Theorem 6.7). This restricts the class of invariants what must be looked for.

7. The non-exact case. To conclude the discussion we consider now the nonexact case. The important point is the following standard result

Proposition 7.1. Given a non-exact contact manifold $(C, D)$. There exists an exact contact manifold $(\hat{C}, \hat{D})$ which is a non-trivial double covering of $C$. The projection is a contactomorphism and it can be found a contact form $\hat{\theta}$ in $\hat{C}$ such that the structure $\mathbb{Z}_{2}$-action of the covering is a strong anti-contactomorphism, i.e. for $\alpha \in \mathbb{Z}_{2}$, it is $\alpha_{*} \hat{\theta}=-\hat{\theta}$.

For a simple proof, see [IMP99]. Now, we follow the ideas in [IMP99]. We lift $S$ to the double covering and denote it again by $S$. It is easy to find an almost complex compatible structure $J$ satisfying that $\alpha_{*} J=-J$, this implies that $\alpha_{*} g_{k}=g_{k}$. The map $\alpha$ lifts to a morphism of bundles $\tilde{\alpha}: L^{\otimes k} \rightarrow \bar{L}^{\otimes k}$ (in fact recalling from [IMP99] that $L$ is trivialized by construction, we take the identity in each fiber). This morphism preserves the connection. So it is easy to check that if $s_{k}$ is a sequence of sections of the bundles $S \otimes L^{\otimes k}$ with $C^{r}$-bounds $\left(c_{D}, c_{R}\right)$ with respect to the contact form $\theta$ and the almost complex structure $J$, then $\alpha_{*} s_{k}$ is a sequence of sections of the bundle $S \otimes \bar{L}^{\otimes k}$ with respect to the contact form $-\theta$ and to the compatible almost complex structure $-J$ with the same mixed $C^{r}$-bounds $\left(c_{D}, c_{R}\right)$. But, now we identify the bundle $L^{\otimes k}$ with $\bar{L}^{\otimes k}$ using the anticomplex isomorphism provided by the identity. Then a simple computation shows that $\alpha_{*} s_{k}$ is a sequence of sections of the bundles $S \otimes L^{\otimes k}$ with the same mixed $C^{3}$-bounds.

Our only task is to assure that all the objects in the construction are $\mathbb{Z}_{2}$-invariant. The precedent considerations assure this condition in the local constructions. Now we are going to study the globalization process. The first point is to achieve the set of points $S$ invariantly. Moreover each $S_{j}$ has to be $\mathbb{Z}_{2}$-invariant. This is only true if the action of $\mathbb{Z}_{2}$ is free, because in any other case we would have problems to assure the second property of the set. The perturbation is performed in a similar way that in the standard case. The key idea is that the perturbation term $\tau_{k, x}$ constructed to obtain transversality close to a point $x$ can be transported to the point $\alpha(x)$ by means of $\tau_{k, \alpha(x)}=\alpha_{*} \tau_{k, x}$. There is no interference between $x$ and $\alpha(x)$, because

$$
d_{k}(x, \alpha(x))=O\left(k^{1 / 2}\right)
$$

The term $\tau_{k, \alpha(x)}$ produces the same transversality as $\tau_{k, x}$ because of the $\mathbb{Z}_{2}$-invariance of the construction.

The perturbation of the function $F^{s_{k}}$ can be made invariantly. In the set $A$ it is clear. In $\Delta$, the trivialization is invariant, as well as the function $H$ and we can choose $w^{\prime}$ invariant without loss of generality.

So we have constructed a pencil in $\hat{C}$ which is $\mathbb{Z}_{2}$-invariant. This $\mathbb{Z}_{2}$-invariance property allows us to quotient by the group $\mathbb{Z}_{2}$ obtaining new data $A=\hat{A} / \mathbb{Z}_{2}, \Delta=$ $\hat{\Delta} / \mathbb{Z}_{2}$ and $f=f / \mathbb{Z}_{2}$. It is a trivial exercise to check that the object so defined is a contact pencil on $C$. 
8. Birational equivalence. A natural question to be thought is how to construct a contact fibration starting with a Lefschetz pencil. The way to follow is to blow-up the base points set to avoid the intersections between different fibers. In the symplectic case this is absolutely standard and tells us that every symplectic manifold is a Lefschetz fibration up to birational equivalence. We will say that two symplectic manifolds are birationally equivalent if they are related through a sequence of symplectic blow-up's and blow-down's. This characterization has allowed S. Donaldson and I. Smith [DSO0] to prove several results concerning symplectic topology.

Our task is to look for a similar argument in the contact case showing that contact manifolds are always "birationally equivalent" to contact fibrations. Two remarks before starting. Firstly, the definition of contact Lefschetz fibration is the natural one: a contact pencil with no base points set. Secondly, we mean by "birational equivalent contact manifolds" two contact manifolds related by a sequence of blowup's and blow-down's. This definition only makes sense if we are able to define what is a contact blow-up.

This notion is not so new, M. Gromov spoke about the construction of contact blow-up's about thirty years ago. Recall that it is easy to define a topological complex blow-up. Suppose we have got a submanifold $N$ inside a smooth manifold $M$ with complex normal bundle. In this case, we blow-up each one of the fibers of this bundle. What we get is a new manifold $\tilde{M}$ called the topological blow-up of $M$. There is a natural projection $\pi: \tilde{M} \rightarrow M$ which is a diffeomorphism in $\tilde{M}-\pi^{-1}(N)$. In the symplectic case it is well-known that if $N$ is a symplectic submanifold then $\tilde{M}$ admits a symplectic structure. It makes sense to "topologically" blow up a contact manifold along a contact submanifold.

The problem is to define a contact structure in the manifold so constructed. In general we have got only the following

CONJECTURE 8.1. Let $(C, D)$ be a contact manifold and let $N$ be a compact contact submanifold. Then there exists a contact structure $\tilde{D}$ in the topological blowup of $C$ along $N$. The contact structure is defined by the pull-back of $D$ through $\pi$ outside an arbitrary small neighborhood of $N$.

We will call quantum contact manifold associated to a symplectic manifold $(M, \omega)$ to the circle bundle $C(M)$ over $M$ with connection $\theta$ whose curvature is the pull-back of $\omega$. The quantum manifold $C(M)$ has got a natural contact structure given by $\theta$. M. Gromov has proved

ThEOREM 8.2 (Exercise (c) pp. 342-343 in [Gr86].). Suppose we have the same hypothesis than in Conjecture 8.1. Also assume that the contact submanifold $N$ is the quantum manifold associated to a symplectic manifold $M$. Moreover, assume that the symplectic normal bundle $\nu(N)$ of $N$ is the pull-back of a symplectic bundle over $M$. Then Conjecture 8.1 holds.

With Theorem 8.2 we can prove some partial results

Corollary 8.3. Every 5-dimensional contact manifold $(C, D)$ is birationally equivalent to a contact Lefschetz fibration with fibers Poincaré dual to any given element of $H^{2}(C)$.

Proof. By Theorem 1.2 the contact manifold has got a contact Lefschetz pencil with the required fibers. To conclude we only need to blow-up the base locus set. In the 5 dimensional case, the set $A$ is a finite set of transverse contact curves. But, obviously, this is the quantum manifold associated to a finite set of points. To satisfy 
the condition of Theorem 8.2, we need only to assure that its normal bundle is trivial. But this is obvious since rank 4 vector bundles over $S^{1}$ are always trivial. Therefore we can blow-up the manifold along $A$ to obtain the required fibration.

\section{REFERENCES}

[Au97] D. Auroux, Asymptotically holomorphic families of symplectic submanifolds, Geom. Funct. Anal., 7 (1997), pp. 971-995.

[Au00] D. Auroux, Symplectic 4-manifolds as branched coverings of $\mathbb{C P}^{2}$, Invent. Math., 139 (2000), pp. 551-602.

Invent. Math., 142 (2000), no. 3, pp. 631-673.

[AK00] D. Auroux, L. KaTZARKOV, Branched coverings of $\mathbb{C P}^{2}$ and invariants of symplectic 4manifolds, Invent. Math., 142 (2000), no. 3, pp. 631-673.

[Bi01] P. BIRAN, Lagrangian barriers and symplectic embeddings, Preprint (2001).

[Co00] V. Colin, Chirurgie de Dehn admissible dans une variété de contact tendue, Preprint (2000).

[Do96] S. K. Donaldson, Symplectic submanifolds and almost-complex geometry, J. Diff. Geom., 44 (1996), pp. 666-705.

[Do98] S. K. Donaldson, Lefschetz fibrations in Symplectic Geometry, Doc. Math. Extra Vol. ICM 98, II, pp. 309-314 (1998).

[Do99] S. K. Donaldson, Lefschetz pencils on symplectic manifolds, J. Differential Geom., 53 (1999), no. 2, pp. 205-236.

[DS00] S. K. Donaldson, I. SmiTh, Lefschetz pencils and the canonical class for symplectic 4manifolds, Preprint. Mathematical Institute, Oxford (2001).

[ET98] Y. Eliashberg, W. Thurston, Confoliations, University Lecture Series, 13, American Mathematical Society, Providence, Rhode Island, (1999).

[Gi91] E. Giroux, Convexité en topologie de contact. Comm. Math. Helvet., 66 (1991), pp. 637677.

[GM01] E. Giroux, J. P. MoHSEn, in preparation.

[Gr76] P. A. GRIFFITHS, Entire holomorphic mappings in one and several complex variables, Ann. Math. Studies n., 85, Princeton University Press, Princeton, 1976.

[Gr86] M. Gromov, Partial Differential relations, Springer-Verlag. Berlin. (1986).

[IMP99] A. Ibort, D. MARTínez, F. Presas, On the construction of contact submanifolds with prescribed topology, J. Diff. Geom., 56 (2000), no. 2, pp. 235-283.

[IMP01] A. IBORT, D. MARTínez, F. PreSAS, Taut and contact structures, in preparation.

[Le24] S. LefsChetz, L'analysis situs et la géométrie algébrique, Gauthier-Villars, Paris, (1924).

[MS94] D. MCDuff., D. SALAMON, Introduction to symplectic topology, Oxford Mathematical Monographs, (1994).

[MPS99] V. MUÑoz, F. Presas, I.Sols, Almost holomorphic embeddings in grassmanians with applications to singular symplectic submanifolds, to appear in J. Reine Angew. Math.

[Pa98] R. PAOLETTI, Symplectic submanifolds over projective fibrations of symplectic manifolds, to appear in Annales de l'Institut Fourier, Grenoble.

[Pa99] R. PAOLETTI, Symplectic submanifolds in special position, preprint. Universita di Pavia. (1999).

[Pr01] F. PRESAS, Submanifolds in symplectic manifolds with contact boundary, preprint. Universidad Complutense de Madrid. (2001).

[Ti77] D. TischleR, Closed 2-forms and an embedding theorem, J. Diff. Geom., 12 (1977), pp. 229-235.

[We91] A. Weinstein, Contact surgery and symplectic handlebodies, Hokkaido Mathematical Journal, 20 (1991), pp. 241-251.

[Yo83] B. YomDin, The geometry of critical and near-critical values of differentiable mappings, Math. Annalen, 104 (1983), pp. 495-515. 
